\title{
Development of a poly(vinyl alcohol)/lysine electrospun membrane-based drug delivery system for improved skin regeneration
}

\author{
Rosa S. Sequeira ${ }^{\mathrm{a}}$, Sónia P. Miguel ${ }^{\mathrm{a}}$, Cátia S.D. Cabral ${ }^{\mathrm{a}}$, André F. Moreira ${ }^{\mathrm{a}}$, Paula Ferreira ${ }^{\mathrm{b}}$, \\ Ilídio J. Correia ${ }^{\mathrm{a}, \mathrm{b}, *}$ \\ ${ }^{\text {a }}$ CICS-UBI - Centro de Investigação em Ciências da Saúde, Universidade da Beira Interior, Av. Infante D. Henrique, 6200-506 Covilhã, Portugal \\ ${ }^{\mathrm{b}}$ CIEPQPF - Departamento de Engenharia Química, Universidade de Coimbra, Rua Silvio Lima, 3030-790 Coimbra, Portugal
}

\section{A R T I C L E I N F O}

\section{Keywords:}

Drug delivery systems

Electrospun membranes

Ibuprofen

Lavender oil

Wound dressing

\begin{abstract}
A B S T R A C T
Nanofiber-based wound dressings are currently being explored as delivery systems of different biomolecules for avoiding skin infections as well as improve/accelerate the healing process. In the present work, a nanofibrous membrane composed of poly(vinyl alcohol) (PVA) and lysine (Lys) was produced by using the electrospinning technique. Further, anti-inflammatory (ibuprofen (IBP)) and antibacterial (lavender oil (LO)) agents were incorporated within the electrospun membrane through blend electrospinning and surface physical adsorption methods, respectively. The obtained results demonstrated that the PVA_Lys electrospun membranes incorporating IBP or LO displayed the suitable morphological, mechanical and biological properties for enhancing the wound healing process. Moreover, the controlled and sustained release profile attained for IBP was appropriate for the duration of the wound healing inflammatory phase, whereas the initial burst release of LO is crucial to prevent wound bacterial contamination. Indeed, the PVA_Lys_LO electrospun membranes were able to mediate a strong antibacterial activity against both $S$. aureus and $P$. aeruginosa, without compromising human fibroblasts viability. Overall, the gathered data emphasizes the potential of the PVA_Lys electrospun membranesbased drug delivery systems to be used as wound dressings.
\end{abstract}

\section{Introduction}

After a skin injury occurs, a complex wound healing process is immediately initiated to re-establish, as soon as possible, skin structure and functions (Gurtner et al., 2008). Up to now, different types of dressing materials (e.g. gauzes, sponges, gels, creams) have been used to cover the wound site, in order to provide protection against external harmful agents, avoid patient dehydration, and promote the skin regeneration process (Pereira et al., 2013). Furthermore, there are gels and creams incorporating drugs $\left(\right.$ Mederma $^{\circledR}$, Contractubex ${ }^{\circledR}$, Cybele ${ }^{\circledR}$ Scagel, Erasé gel, etc) that are used in the clinic to accelerate/ ameliorate the wound healing process (Gizaw et al., 2018; Miguel et al., 2018; Sidgwick et al., 2015). However, these dressings require periodic replacement and display a limited therapeutic outcome. Additionally, the frequent wound management can often be painful for the patient as well as cost- and labour-intensive for the healthcare system (Gizaw et al., 2018; Miguel et al., 2018). To surpass these handicaps, researchers have been devoted to develop advanced regenerative dressings, that: i) protect the wound; ii) are biodegradable; iii) are biocompatible; iv) promote the wound exudate absorption and v) are able to provide a sustained release of drugs that enhance the wound healing process as well as decrease patient pain (Gizaw et al., 2018; Miguel et al., 2018; Miguel et al., 2019a).

Electrospun nanofiber-based wound dressings have arisen as one of the most promising therapeutic solutions for wound healing management, since they exhibit structural features that are quite similar to those presented by the native skin's extracellular matrix (ECM) (Miguel et al., 2017; Miguel et al., 2018; Miguel et al., 2019b; Thakkar and Misra, 2017). Moreover, they present a high surface-to-volume ratio that provides more space for cell attachment as well as a tuneable porosity that allows the control over the wound site humidity and nutrients exchange (Miguel et al., 2018). Further, the excellent mechanical properties exhibited by electrospun membranes guarantee that they can endure the mechanical forces during its handling and the regeneration process (Akduman et al., 2016; Chou et al., 2015; Thakkar and Misra, 2017). Additionally, electrospun nanofibers can act as drug delivery systems (Akduman et al., 2016; Braghirolli et al., 2014; Miguel et al., 2019a) that exhibit efficient/sustained drug release profiles,

\footnotetext{
*Corresponding author at: CICS-UBI - Centro de Investigação em Ciências da Saúde, Universidade da Beira Interior, Avenida Infante D. Henrique, 6200-506 Covilhã, Portugal.

E-mail address: icorreia@ubi.pt (I.J. Correia).
} 
leading to a reduction in the frequency of the treatments' topical application. Besides that, drug incorporation on nanofibers can be achieved through different methods (e.g. blend, co-axial, physical adsorption, and chemical immobilization) rendering them the capacity to mediate the delivery of different biomolecules with specific release profiles (Im et al., 2010; Miguel et al., 2018; Miguel et al., 2019a). The physical adsorption method is commonly associated with burst releases, while the co-axial electrospinning enables a more controlled and sustained release profile (Im et al., 2010; Zamani et al., 2013). Sergio Torres-Giner and colleagues verified that the coaxial PLA-collagen fibers unveiled a more controlled release profile of gentamicin ( $41 \%$ after $24 \mathrm{~h}$ ) than the blend PLA-collagen fibers (98\% of gentamicin was released after $24 \mathrm{~h}$ ) (Torres-Giner et al., 2012). On the other side, Monteiro et al. observed that the release profile of gentamicin covalently immobilized at the surface of chitosan nanofibers meshes showed an initial burst release (about $1 \mathrm{mg}$ of gentamicin was released after $10 \mathrm{~h}$ of incubation) (Monteiro et al., 2015). In this field, there are several reports addressing the electrospun membranes applying different methodologies to incorporate antimicrobial (e.g. antibiotics, silver-based materials, and natural extracts) and biological (e.g. growth factors, vitamins and anti-inflammatory drugs) molecules, which will allow to obtain distinct release profiles, as extensively reviewed by Miguel et al. (2019a).

Herein, a multifunctional electrospun membrane able to incorporate both biological (an anti-inflammatory drug) or antimicrobial (a plant extract) compounds was produced. To accomplish that, Poly(vinyl alcohol) (PVA) and Lysine (Lys) were combined. The PVA is a synthetic polymer used in different tissue engineering applications, due to its hydrophilicity, good chemical resistance, biocompatibility, viscoelasticity and excellent mechanical performance (Jatoi et al., 2019; Majd et al., 2016). In recent works, the applicability of the PVA-based biomaterials in drug delivery and wound healing applications has been demonstrated (Mohseni et al., 2019; Saviano et al., 2019; Shamloo et al., 2018). In turn, Lys is an aminoacid that can promote cell adhesion and proliferation as well as tissue regeneration at the biomaterial interface. In the biological media, the amine groups available on Lys molecule are protonated, thus facilitating its interaction with the negatively-charged cell membrane (Mekhail et al., 2014). In a previous study, Mahanta and Valiyaveettil produced electrospun PVA membranes chemically modified with Lys to be applied in the extraction of nanosized contaminants from the aqueous environment (Mahanta and Valiyaveettil, 2011). Moreover, Amariei and co-workers incorporated the antimicrobial polypeptide $\varepsilon$-poly(l-lysine) ( $\varepsilon$-PL) into poly(acrylic acid) (PAA)/PVA electrospun membranes through electrostatic interactions to create a wound dressing (Amariei et al., 2018). However, in this work, the Lys and PVA were combined in a polymeric solution, before the electrospinning process be performed, to improve the biological properties of the electrospun membranes. Furthermore, to obtain distinct drug release profiles, different drug incorporation methods were used in order to tailor the action of each biomolecule to a specific time frame. For instance, the inflammation phase occurs 1-3 days after injury, and consequently, the release profile of anti-inflammatory drugs must be adjusted to this time-frame (Hart, 2002; Morgado et al., 2014). On the other hand, the antimicrobial molecules should be released to the wound site immediately after the dressing application to avoid any bacterial contamination (Guo and Di Pietro, 2010; Simões et al., 2018b). In this study, it was studied and optimized the release profile of an anti-inflammatory molecule (ibuprofen (IBP), which was entrapped in the nanofibers through blend electrospinning, and an antimicrobial agent (Lavender oil (LO)), which was incorporated in the nanofibers by physical adsorption. IBP is a non-steroidal anti-inflammatory drug (NSAID) that inhibit the two isoforms of cyclooxygenase (COX-1 and COX-2), which are involved in the biosynthesis of prostaglandins (proinflammatory molecules) (Morgado et al., 2014; Morgado et al., 2017). The anti-inflammatory performance of IBP was previously evaluated in full-thickness wounds by Mohiti-Asli et al., which reported that the PLA nanofibrous membranes containing $20 \%$ of IBP supported the wound closure (60\% wound contraction after 14 days) (Mohiti-Asli et al., 2017). On the other side, the LO is one natural product extracted from lavender plant species such as Lavandula latifolia, Lavandula stoechas, and Lavandula angustifolia, displaying excellent antimicrobial and antioxidant properties (Balasubramanian and Kodam, 2014; Danh et al., 2012; Predoi et al., 2018).

The experimental data gathered in this study supports the application of the PVA_Lys multifunctional electrospun membrane as a temporary support for cell attachment, proliferation, and hence the skin regeneration process. In addition, the release profiles of the anti-inflammatory drug (IBP) and the antimicrobial compound (LO) obtained herein are suitable to achieve an enhanced healing process.

\section{Material and methods}

\subsection{Materials}

Fetal bovine serum (FBS) free from any antibiotic was purchased from Biochrom AG (Berlin, Germany). Normal human dermal fibroblasts (NHDF) cells were acquired from PromoCell (Labclinics, S.A., Barcelona, Spain). 3-(4,5-dimethylthiazol- 2-yl)-5-(3-carboxymethoxyphenyl)-2 (4-sulfophenyl)-2H-tetrazolium (MTS) was bought from Promega (Madison, WI, USA). L-lysine (98\%, Mw $=146,19 \mathrm{~g} / \mathrm{mol}$ ) was obtained by Acros organics (China). Ibuprofen (IBP) was bought by TCI (Tokyo Chemical Industry. Lavender oil (LO) was acquired by Keypharm (Belgium). Dulbecco's modified eagle's medium (DMEMF12), Poly(ethylene oxide) ( $\mathrm{Mw}=300,000 \mathrm{~g} / \mathrm{mol})$, Poly(vinyl alcohol) $(\mathrm{Mw}=89,000-98,000 \mathrm{~g} / \mathrm{mol}) \quad 99 \%$ hydrolyzed, ethylenediaminetetraacetic acid (EDTA), glutaraldehyde, LB Broth, phosphate-buffered saline solution (PBS), 2,2-diphenly-1-picrylhydrazyl (DPPH), Triton X100 and trypsin were purchased from Sigma-Aldrich (Sintra, Portugal). Quant-iT Pico Green dsDNA assay kit and Hoechst $33342^{\circledR}$ were obtained from ThermoFisher Scientific (Waltham, MA, USA). Staphylococcus aureus clinical isolate (S. aureus) ATCC 25923 and Pseudomonas aeruginosa ( $P$. aeruginosa) obtained from a human sample were used as models of prokaryotic organisms to evaluate the bactericidal activity exhibited by the produced membranes. Propidium iodide buffer and WGA-Alexa $594^{\circledR}$ conjugate were gotten from Invitrogen (Carlsbad, California, EUA) and Calcein AM was supplied by Calbiochem (Merck Millipore, Oeiras, Portugal). A Milli-Q Advantage A10 ultrapure Water Purification System $(0.22 \mu \mathrm{m}$ filtered; $18.2 \mathrm{M} \Omega$ / $\mathrm{cm}$ at $25^{\circ} \mathrm{C}$ ) was used to obtain double deionized and filtered water.

\subsection{Methods}

\subsubsection{Production of electrospun membranes}

The electrospun membranes were produced by using a conventional electrospinning apparatus. This equipment was composed by a high voltage source (Spellman CZE1000R, 0-30 kV), a precision syringe pump (KDS-100), a plastic syringe with a stainless-steel needle (21 Gauge), and an aluminum foil connected to a copper collector.

Initially, a PVA solution 9\% (w/v) was dissolved in Milli-Q water in $90^{\circ} \mathrm{C}$ under stirring for $1 \mathrm{~h}$. Subsequently, the temperature was reduced to $60{ }^{\circ} \mathrm{C}$ and then the L-lysine, at a concentration of $12.5 \mathrm{mg} / \mathrm{mL}$, was added to the solution and mixed under magnetic stirring for $30 \mathrm{~min}$. Lastly, $2 \mathrm{~mL}$ of PEO solution ( $8 \% \mathrm{w} / \mathrm{v}$ ) were added to obtain a solution viscosity that is suitable for the electrospinning process. After that, $10 \mathrm{~mL}$ of the solution were loaded into the syringe, and electrospun using a constant flow rate $(1 \mathrm{~mL} / \mathrm{h})$, a working distance of $12 \mathrm{~cm}$, and an applied voltage of $30 \mathrm{kV}$, resulting in PVA_Lys membrane.

In turn, the PVA_Lys_IBP membrane was obtained through the addition of IBP (at $1 \mathrm{mg} / \mathrm{mL}$ ) to the PVA_Lys solution before the electrospinning process. On the other side, the LO was adsorbed to the surface of the PVA_Lys membrane by exploring the electrostatic interactions that occur between the positively charged amine groups of Lys and the 
electronegative groups (e.g. acetate, aldehyde, and terpenoid moieties) found in LO. For that purpose, $15 \mu \mathrm{L}$ of $\mathrm{LO}(1.3 \mathrm{mg} / \mathrm{mL}$ in ethanol (99.99\%)) were placed at the PVA_Lys membrane' surface $\left(0.79 \mathrm{~cm}^{2}\right)$ and incubated at $50{ }^{\circ} \mathrm{C}$, under vacuum, during $1 \mathrm{~h}$, yielding to PVA_Lys_LO membrane. The IBP and LO concentrations were selected based on the cell viability data (please see Fig. S1).

\subsubsection{Characterization of the solutions' properties and membranes' morphology}

The viscosity of polymer solutions (PVA_Lys, PVA_Lys_PEO, and PVA_Lys_PEO_IBP) were measured by using a rotational viscometer (Brookfield, DVT3TRVCP), using a spindle speed of $20 \mathrm{rpm}$ at $27.7^{\circ} \mathrm{C}$. A $\mathrm{CP}-40$ cone-plate measuring system was used. All rheological measurements were performed in triplicate to ensure reproducibility.

In turn, the conductivity of polymeric solutions $(n=3)$ was determined by using a conductivity meter (FiveEasy Cond meter F30, Mettler Toledo, Malaysia), at $27.7^{\circ} \mathrm{C}$ (Son et al., 2004). The solutions' surface tension values were determined by using a PC controlled KSV Sigma 70 tension balance, which employs the Du-Noüy ring detachment method taking into account the Huh and Mason correction for interface distortion.

Further, the produced membranes were also analyzed by Scanning electron microscopy (SEM) to characterize their morphology and the fibers' diameter. To accomplish that, the samples were assembled onto aluminum stubs using Araldite glue and sputter-coated with gold using a Quorum Q150R ES sputter coater (Quorum Technologies Ltd., Laughton, East Sussex, UK). The SEM images were obtained using the Hitachi S-3400 N Scanning Electron Microscope (Hitachi, Tokyo, Japan), at an accelerating voltage of $20 \mathrm{kV}$. The nanofibers' diameters were measured using an image analysis software - Image $\mathrm{J}$ (Scion Corp., Frederick, MD) (Simões et al., 2018a).

\subsubsection{Attenuated total reflectance-Fourier transform infrared spectroscopy analysis}

To characterize the chemical composition of the membranes, attenuated total reflectance-Fourier transform infrared spectroscopy (ATRFTIR) analysis was used. The membranes' spectra were obtained with an average of 128 scans, a spectral width ranging from 4000 and $400 \mathrm{~cm}^{-1}$ and a spectral resolution of $32 \mathrm{~cm}^{-1}$, using a Nicolet iS10 FTIR spectrophotometer (Thermo Scientific, Waltham, MA, USA). In addition, the membranes raw materials were also subjected to spectral analysis for comparative purposes.

\subsubsection{Characterization of the mechanical properties of the membranes}

The mechanical properties of the electrospun membranes were evaluated using a Shimadzu AG-X Tensile Testing Machine (Tokyo, Japan), at room temperature (RT), under wet and dry conditions, accordingly to the guidelines established by the Standard Test Method for Tensile Properties of Polymer Matrix Composite Materials (ASTM standard D3039/D3039 M) (Morgado et al., 2014).

To perform this assay, samples $(\mathrm{n}=5)$ with a width of $2 \mathrm{~cm}$, gauge length of $6 \mathrm{~cm}$ and thickness ranging from 0.41 to $0.52 \mathrm{~mm}$ were used. The length between the clamps was set to $2 \mathrm{~cm}$ and the speed of testing was set to $20 \mathrm{~mm} / \mathrm{min}$. For the wet conditions, the membranes were previously immersed in a PBS solution $(\mathrm{pH}=5.5)$, over $2 \mathrm{~h}$ and at $37^{\circ} \mathrm{C}$. Load-extension data was recorded and the membranes' stress-strain curve was obtained through the Eqs. (1) and (2), respectively:

Stress $=\sigma=\frac{F}{A}$

Strain $=\frac{\Delta l}{L}$

where $\mathrm{F}$ is the applied force; $\mathrm{A}$ is the cross-sectional area; $\Delta \mathrm{l}$ is the change in length, and $\mathrm{L}$ is the length between the clamps.

\subsubsection{Determination of the membranes' porosity}

The total porosity of the membranes was evaluated by using a liquid displacement method described elsewhere (Miguel et al., 2017). For this assay, five specimens were weighed and then immersed in absolute EtOH during $1 \mathrm{~h}$. Then, the samples were re-weighted and the membranes' overall porosity was determined through Eq. (3):

Porosity $(\%)=\frac{W_{s}-W_{d}}{D_{\text {ethanol }} \times V_{\text {membrane }}} \times 100$

where $\mathrm{W}_{\mathrm{s}}$ and $\mathrm{W}_{\mathrm{d}}$ represent the swollen and dry weights of the membrane, respectively. The $\mathrm{D}_{\text {ethanol }}$ is the density of the ethanol at RT and $\mathrm{V}_{\text {membrane }}$ is the volume of the swollen membrane.

\subsubsection{Evaluation of the membranes' wettability}

The wettability of the membranes was characterized by determining the water contact angle (WCA) at their surfaces. To accomplish that, the WCA was measured using the Data Physics Contact Angle System OCAH 200 apparatus, operating in static mode, at RT (Miguel et al., 2019b). This assay consisted on the deposition of water drops $(4 \mu \mathrm{L})$ at different locations of the electrospun membranes' surface $(n=5)$.

\subsubsection{Water vapor transmission rate (WVTR)}

The water vapor diffusion through the membranes was determined as reported elsewhere (Antunes et al., 2015). Briefly, the membranes $(n=5)$ were used to seal the opening of a glass test tube $\left(1.77 \mathrm{~cm}^{2}\right.$ ) containing $10 \mathrm{~mL}$ of ultrapure water. To prevent non-specific water evaporation, parafilm tape was used to fix the membrane in a glass tube. Posteriorly, the membranes were incubated at $37^{\circ} \mathrm{C}$ and the amount of evaporated water was measured by monitoring the weight variations at specific time points. Then, the WVTR was obtained through Eq. (4):

Water Vapor Transmission Rate $=\frac{W_{\text {loss }}}{A}$

where $\mathrm{W}_{\text {loss }}$ is the daily weight loss of water and $A$ is the area of the tube opening.

\subsubsection{Evaluation of the membranes' swelling capacity}

The swelling capacity of the produced membranes was determined by immersing them in a PBS solution at $\mathrm{pH} 5$ (pH of native skin) or $\mathrm{pH} 8$ ( $\mathrm{pH}$ displayed by injured skin), at $37^{\circ} \mathrm{C}$ under stirring ( $40 \mathrm{rpm}$ ) (Miguel et al., 2019b; Simões et al., 2018a). After, the excess of PBS was removed with a filter paper, the samples $(n=5)$ were weighted. In turn, the swelling ratio was calculated using Eq. (5):

Swelling ratio $(Q)=\frac{W_{t}}{W_{0}}$

where $W_{t}$ is the final weight and $W_{0}$ is the initial weight of the membranes.

\subsubsection{Characterization of the membranes' biodegradation profile}

The degradation profile of the membranes was monitored by immersing the samples in PBS, under stirring ( $40 \mathrm{rpm})$, at $37^{\circ} \mathrm{C}$. After 1,3 and 7 days, the membranes $(n=5)$ were removed from the solutions, fully dried and weighed. Then, the degradation percentage was calculated at each time through Eq. (6):

Weight loss $(\%)=\frac{W_{i}-W_{t}}{W_{i}} \times 100$

where $\mathrm{W}_{\mathrm{i}}$ corresponds to the initial weight of the sample and $\mathrm{W}_{\mathrm{t}}$ to the weight of the sample at time $t$.

\subsubsection{Evaluation of the membranes' antioxidant capacity}

The antioxidant activity of the membranes was evaluated through the 2-diphenly-1-picrylhydrazyl (DPPH) radical scavenging assay, as previously described in the literature (Miguel et al., 2019b; Simões 
et al., 2018a). To perform this assay, membrane samples ( $5 \mathrm{mg}$; $\mathrm{n}=5$ ) were immersed in $3 \mathrm{~mL}$ of DPPH solution $(100 \mu \mathrm{M})$ in methanol and incubated in a dark room, at RT during $30 \mathrm{~min}$. Afterward, the absorbance of each mixture was recorded using a microplate reader (Biorad xMark microplate spectrophotometer), at $517 \mathrm{~nm}$. The antioxidant activity was then calculated using the Eq. (7):

Antioxidant activity $(\%)=\frac{A_{c}-A_{s}}{A_{c}} \times 100$

where $A_{c}$ and $A_{s}$ represent the control and sample absorbance values, respectively.

\subsubsection{Evaluation of the IBP and $L O$ encapsulation and loading efficiencies}

The study of the IBP and LO encapsulation and loading efficiencies was performed according to the method described by Miguel et al. (2019b). The PVA_Lys_IBP and PVA_Lys_LO membranes $(n=5)$ were subjected to total degradation, through their immersion in hot water, under sonication, for $2 \mathrm{~h}$. Then, the samples were centrifuged at 18000 $g$ for $10 \mathrm{~min}$ in order to obtain the supernatant. The supernatant absorbance was recorded using a Thermo Scientific Evolution 201 UV-vis spectrophotometer, at 264 and $223 \mathrm{~nm}$, for IBP and LO, respectively. After that, the IBP and LO concentrations were determined using standard calibration curves for IBP $(\mathrm{y}=1.4263 \mathrm{x}+0.0196$, $\left.\mathrm{R}^{2}=0.999\right)$ and for LO $\left(\mathrm{y}=1.3833 \mathrm{x}+2.6952, \mathrm{R}^{2}=0.996\right)$. The encapsulation efficiency (EE) and loading efficiency (LE) for both bioactive agents were determined using Eqs. (8) and (9) (Moreira et al., 2014):

$E E(\%)=\frac{W_{i}-W_{s}}{W_{i}} \times 100$

$L E(\%)=\frac{W_{i}-W_{s}}{Y} \times 100$

where $\mathrm{W}_{\mathrm{i}}$ is the total amount of IBP or LO added for loading, $\mathrm{W}_{\mathrm{s}}$ is the amount of compound in the supernatant, and $\mathrm{Y}$ is the total dry weight of PVA_Lys_IBP and PVA_Lys_LO membranes.

\subsubsection{In vitro IBP and $L O$ release profile and analysis of the drug-release} kinetics

The amount of IBP and $\mathrm{LO}$ released at two $\mathrm{pHs}$ values $(\mathrm{pH} 5$ and $\mathrm{pH}$ 8) was determined following the protocols already described in the literature (Balasubramanian and Kodam, 2014; Miguel et al., 2019b; Morgado et al., 2014). Briefly, the PVA_Lys_IBP and PVA_Lys_LO membranes $(15 \mathrm{mg})$ were incubated at $37^{\circ} \mathrm{C}$ under stirring $(40 \mathrm{rpm})$ with $1 \mathrm{~mL}$ of PBS at pH 5 and $8(n=5)$. Then, at specific timepoints, the samples were centrifugated (at $18,000 \mathrm{~g}$, for $10 \mathrm{~min}$ ) and the supernatant' absorbance was measured.

\subsubsection{Characterization of the biological properties of the produced membranes}

2.2.13.1. Evaluation of cell viability and proliferation in contact with electrospun membranes. The membranes' biocompatibility was characterized through an MTS assay, according to the ISO 10993-5:2009 (Biological evaluation of medical devices- Part 5: Tests for in vitro cytotoxicity). To accomplish that, the produced membranes $(n=5)$ with sizes inferior to $10 \%$ of the well area, were placed into 96 well plates and then sterilized under UV irradiation $(254 \mathrm{~nm}, \approx 7 \mathrm{~mW}$ $\mathrm{cm}^{-2}$ ) during $1 \mathrm{~h}$. Then, NHDF cells were seeded (cell passage between 15 and 20) at a density of $10 \times 10^{3}$ cells per well and incubated at $37^{\circ} \mathrm{C}$, in an incubator with a $5 \% \mathrm{CO}_{2}$ humidified atmosphere. After 1,3 , and 7 days of incubation, the medium of each well was removed and replaced by a mixture of $100 \mu \mathrm{L}$ of fresh culture medium and $20 \mu \mathrm{L}$ of MTS/PMS (phenazine methosulfate) reagent solution. Following $4 \mathrm{~h}$ of incubation (at $37^{\circ} \mathrm{C}$, in a $5 \% \mathrm{CO}_{2}$ atmosphere), the samples' absorbance was measured at $490 \mathrm{~nm}$ using a microplate reader (Biorad xMark microplate spectrophotometer). Further, the positive control $\left(\mathrm{K}^{+}\right)$and negative control $\left(\mathrm{K}^{-}\right)$were also used, which correspond to the cells incubated with ethanol (70\%) and cells incubated only with culture medium, respectively.

2.2.13.2. dsDNA quantification. The NHDF proliferation in contact with the membranes was also evaluated by measuring the dsDNA content using a Quant-iT PicoGreen dsDNA Assay kit, as reported in the literature (Miguel et al., 2017; Miguel et al., 2019b; Simões et al., 2018a). Initially, the cells were placed in contact with the produced membranes $(\mathrm{n}=5)$ and after 1,3 and 7 days of incubation, the cell lysis was induced by adding Triton X-100 $(1 \times)$ to the wells during $1 \mathrm{~h}$. Then, the membranes were transferred into a $1.5 \mathrm{~mL}$ Eppendorfs and subjected to a freeze-thaw cycle. Posteriorly, the samples were sonicated and centrifuged at $18,000 \mathrm{~g}$, for $15 \mathrm{~min}$, to obtain the supernatant. After, $100 \mu \mathrm{L}$ of supernatant was added to an equal volume of PicoGreen solution in TE buffer $(1 \times)$. Lastly, the plate was incubated in the dark for $10-15 \mathrm{~min}$ and the fluorescence was measured at excitation and emission wavelengths of $485 \mathrm{~nm}$ and $535 \mathrm{~nm}$ using a microplate reader. To quantify the dsDNA concentrations, a calibration curve was determined using samples with known concentrations of dsDNA.

2.2.13.3. Live/dead assay. The presence of viable and dead cells on the produced membranes was monitored by using the Live/Dead assay (Miguel et al., 2019b; Simões et al., 2018a). For that purpose, cells $\left(10 \times 10^{3}\right.$ cells $\left./ \mathrm{mL}\right)$ were seeded in contact with membranes $(\mathrm{n}=3)$, in $\mu$-Slide 8 well Ibidi imaging plates (Ibidi GmbH, Germany). Cells seeded only with culture medium were used as the negative control $\left(\mathrm{K}^{-}\right)$, while those treated with Triton $\mathrm{X}-100$ were used as the positive control $\left(\mathrm{K}^{+}\right)$. After 1, 3 and 7 days, samples were stained with Calcein $(2 \mu \mathrm{M})$ and Propidium iodide $(15 \mathrm{mM})$, following the instructions of the manufacturer. Then samples were imaged with a confocal laser scanning microscope (CLSM, Zeiss, Oberkochen, Germany) and processed in the Zeiss Zen 2010 software (Zeiss, Oberkochen, Germany).

2.2.13.4. Characterization of cell proliferation through confocal microscopy analysis. The cell distribution and proliferation at the electrospun membranes' surface was evaluated by CLSM. To perform that, cells $\left(10 \times 10^{3}\right.$ cells $\left./ \mathrm{mL}\right)$ were seeded in $\mu$-Slide 8 well Ibidi imaging plates (Ibidi $\mathrm{GmbH}$, Germany) in contact with membranes $(\mathrm{n}=3)$. After 1 and 3 days of incubation, the cells were stained with the WGAAlexa $594^{\circledR}$ conjugate $(5 \mu \mathrm{M})$ and Hoechst $33342^{\circledR}(2 \mu \mathrm{M})$, to label the cytoplasm (red) and cell nucleus (blue), respectively (Figueira et al., 2016). The 3D reconstruction and the image analysis were performed using Zeiss Zen 2010 software (Zeiss, Oberkochen, Germany).

\subsubsection{Evaluation of the produced membranes' antimicrobial activity}

Membranes' antibacterial performance was studied through the determination of the inhibition area produced by the membranes when in contact with $S$. aureus or $P$. aeruginosa (Miguel et al., 2017; Miguel et al., 2019b). Briefly, $200 \mu \mathrm{L}$ of bacteria medium containing $1 \times 10^{8}$ $\mathrm{CFU} / \mathrm{mL}$ of $S$. aureus or $P$. aeruginosa were inoculated in LB agar plates. Then, circular samples of the membranes $(1 \mathrm{~cm}$ of diameter; $\mathrm{n}=3$ ) were placed in the plate and then incubated for $24 \mathrm{~h}$, at $37^{\circ} \mathrm{C}$. The percentage of inhibition area was calculated using Eq. (10):

Inhibition area $(\%)=\frac{A_{i}-A_{0}}{A_{0}}$

where, $A_{i}$ and $A_{0}$ represent the inhibition area and the area of the membranes' disc, respectively.

In addition, the antibacterial effect of the membranes against $S$. aureus and $P$. aeruginosa was also evaluated by quantifying the colonyforming units. Briefly, $50 \mathrm{mg}$ of each membrane sample $(\mathrm{n}=3)$ was added to $10 \mathrm{~mL}$ of LB broth containing $1 \times 10^{5} \mathrm{CFU} / \mathrm{mL}$ of early mid$\log$ phase bacteria culture and then incubated at $37^{\circ} \mathrm{C}$, for $24 \mathrm{~h}$. After 
the incubation period, serial dilutions were prepared and $100 \mu \mathrm{L}$ of bacterial samples were transferred into LB agar plates and incubated for $24 \mathrm{~h}$, at $37^{\circ} \mathrm{C}$. The bacterial colonies were then counted and defined as $\mathrm{CFU} / \mathrm{mL}$ (Miguel et al., 2017; Miguel et al., 2019b). The antibacterial efficiency of the membranes was calculated through Eq. (11):

Antibacterial efficiency $(\%)=\frac{N_{0}-N}{N_{0}} \times 100$

where $\mathrm{N}_{0}$ and $\mathrm{N}$ represent the $\mathrm{CFU} / \mathrm{mL}$ of the control and the experimental group, respectively.

\subsubsection{Characterization of biological properties of the membranes through SEM analysis}

The cellular adhesion and biofilm formation at the PVA_Lys, PVA_Lys_IBP and PVA_Lys_LO membranes' surface $(n=3)$ were characterized through SEM analysis. Initially, samples containing cells or bacteria were fixed with $2.5 \%(\mathrm{v} / \mathrm{v})$ glutaraldehyde for $15 \mathrm{~min}$ and $30 \mathrm{~min}$, respectively. Then, samples were frozen during $1-2 \mathrm{~h}$ and freeze-dried for $3 \mathrm{~h}$ (for samples containing cells) and $6 \mathrm{~h}$ (for samples with bacteria) (Cabral et al., 2019; Miguel et al., 2019b). Lastly, the samples were prepared for SEM analysis, according to the protocol previously described in Section 2.2.2.

\subsubsection{Statistical analysis}

The statistical analysis of the obtained results was performed using one-way analysis of variance (ANOVA), with the Newman-Keuls post hoc test. A p value lower than 0.05 ( $\mathrm{p}<0.05$ ) was considered statistically significant.

\section{Results and discussion}

\subsection{Characterization of the morphological and physicochemical properties of produced nanofibrous membranes}

The advances in the nanotechnology area highlighted the promising properties of the electrospun nanofibers to be used as a drug delivery systems (Goyal et al., 2016; Miguel et al., 2019a). Indeed, the application of the nanofibrous carriers allows a site-specific release of the drugs, reducing the total drug concentration required to exert the therapeutic effect, as well as minimize the associated side effects (Chou et al., 2015). Herein, a PVA_Lys electrospun membrane-based drug delivery system was produced, in which ibuprofen (IBP) or lavender oil (LO) were incorporated, by using blend electrospinning and surface physical adsorption, respectively. The viscosity, electrical conductivity and surface tension of the polymeric solutions were determined (for further details see Table 1). As expected, the PEO addition improved all parameters, making possible the electrospinning of the PVA_Lys solution. On the other side, the IBP incorporation promoted a decrease on the viscosity, electrical conductivity and surface tension values of the blend solution.

In addition, the morphological properties of the produced membranes were investigated through SEM analysis and the fibers' diameter were assessed by performing SEM images analysis using ImageJ software (as seen in Fig. 1). The obtained results showed that the electrospun membranes displayed a randomly oriented mesh composed of highly interconnected fibers with a bead-free smooth surface.

Table 1

Determination of the viscosity and electrical conductivity of polymeric solutions used in the electrospinning process.

\begin{tabular}{llll}
\hline $\begin{array}{l}\text { Polymeric } \\
\text { solutions }\end{array}$ & Viscosity (mPa.s) & $\begin{array}{l}\text { Electrical conductivity } \\
(\mu \mathrm{S} / \mathrm{cm})\end{array}$ & $\begin{array}{l}\text { Surface Tension } \\
(\mathrm{mN} / \mathrm{m})\end{array}$ \\
\hline PVA_Lys & $59.53 \pm 2.19$ & $943.47 \pm 38.55$ & $62.09 \pm 0.13$ \\
PVA_Lys_PEO & $311.97 \pm 6.49$ & $1770.75 \pm 38.48$ & $60.94 \pm 0.06$ \\
PVA_Lys_IBP & $222.37 \pm 1.18$ & $1315.75 \pm 114.79$ & $54.59 \pm 0.05$ \\
\hline
\end{tabular}

Moreover, the PVA_Lys, PVA_Lys_IBP, and PVA_Lys_LO fibers had a mean diameter value of $474.22 \pm 144.85 \mathrm{~nm}, 385.03 \pm 108.21 \mathrm{~nm}$ and $487.14 \pm 155.81 \mathrm{~nm}$, respectively. Such results show that that the incorporation of IBP promotes a decrease on the fibers' diameter when compared with PVA_Lys membrane, while the LO inclusion did not induce any noticeable changes on the fibers' size. Such differences may be related to the method used for the therapeutics loading (blend or surface adsorption), the establishment of hydrophobic interactions between the IBP and the PVA_Lys, or even by the decrease on the solution viscosity, surface tension, and electrical conductivity upon the IBP incorporation (Tungprapa et al., 2007; Zeng et al., 2003). Similar results were previously reported by Pires et al., who observed a decrease in the poly(trimethylene carbonate-co- $\varepsilon$-caprolactone) nanofibers diameter from $1.09 \pm 0.10 \mu \mathrm{m}$ (for non-loaded fibers) to $0.84 \pm 0.08 \mu \mathrm{m}$ (for fibers loaded with IBP 5\%) (Pires et al., 2016). In a similar way, Liu et al. also noticed that the mean fiber diameter decreased by raising the IBP concentration, ie, $1.45 \pm 0.71 \mathrm{~mm}$ (without IBP), $1.40 \pm 0.52 \mathrm{~mm} \quad(2 \% \quad$ IBP $), \quad 1.32 \pm 0.67 \mathrm{~mm} \quad(6 \% \quad$ IBP $)$ and $1.25 \pm 0.59 \mathrm{~mm}$ (10\% IBP) (Liu et al., 2012).

On the other side, the incorporation of LO into PVA_Lys nanofibers through the surface physical adsorption technique allowed the production of the PVA_Lys_LO membrane displaying a more irregular and rougher surface. This methodology has been previously used to immobilize growth factors and heparin on the nanofibers' surface without inducing significant differences on the nanofibers morphology (Rajangam et al., 2006). Additionally, it is worth to notice that all the electrospun membranes presented nanofibers with diameters that are within the range of collagen fibrils found at the ECM $(50-500 \mathrm{~nm})$, increasing its structural similarity with the native skin tissue (Figueira et al., 2016; Miguel et al., 2017; Miguel et al., 2018).

Furthermore, the chemical composition of the membranes was assessed through ATR-FTIR analysis. The spectra of raw materials used for membranes' production are presented in Fig. 2A. The PVA spectrum presents its characteristics peaks at $3299 \mathrm{~cm}^{-1}$ (O-H stretching), $2917 \mathrm{~cm}^{-1}$ (asymmetric $\mathrm{CH}_{2}$ stretching), $1427 \mathrm{~cm}^{-1}$ (CH-O-H stretching), $1085 \mathrm{~cm}^{-1}$ (C-O stretching), and $838 \mathrm{~cm}^{-1}$ (C-C stretching) (Najafi et al., 2018; Santos et al., 2014). In turn, the Lys amide (I) and amide (II) vibrations are clearly visible at 1572 and $1511 \mathrm{~cm}^{-1}$, respectively (Zhai et al., 2002; Zhao et al., 2003). Further, it is also possible to observe the characteristic band $\left(\mathrm{CH}_{2}\right)$ of PEO in the region between $2900 \mathrm{~cm}^{-1}$ and $2850 \mathrm{~cm}^{-1}$ (Miguel et al., 2017). On the other side, Fig. S2 displays the characteristic peaks of the compounds incorporated into PVA_Lys membranes. The spectrum of IBP evidenced its two characteristic peaks: at $2941 \mathrm{~cm}^{-1}$ (OH bending) and at $1703 \mathrm{~cm}^{-1}$ (C = O stretching) (Carreras et al., 2013; Yu et al., 2009). In turn, the $\mathrm{LO}$ spectrum presents a broad peak at $3457 \mathrm{~cm}^{-1}(\mathrm{O}-\mathrm{H}$ stretch, indicating the presence of alcohol); at $2963 \mathrm{~cm}^{-1}(\mathrm{C}=\mathrm{C})$, $2924 \mathrm{~cm}^{-1}$ (stretching of methyl groups); strong bands at $1732 \mathrm{~cm}^{-1}$ $(\mathrm{C}=\mathrm{O}), 1371 \mathrm{~cm}^{-1}$ and $1246 \mathrm{~cm}^{-1}$ (C-O) of ester groups. In addition, it is possible to see peaks at $1008 \mathrm{~cm}^{-1}, 919 \mathrm{~cm}^{-1}, 833 \mathrm{~cm}^{-1}$ and $609 \mathrm{~cm}^{-1}$, which can be assigned to LO C-H deformations (Balasubramanian and Kodam, 2014; Morgan et al., 2006).

Through the analysis of the spectra of the membranes (Fig. 2B), it is noticeable the presence of the characteristic peaks of its raw materials (PVA, Lys, PEO). Furthermore, the peaks of IBP and LO can also be observed in the spectrum of PVA_Lys_IBP and PVA_Lys_LO membranes, respectively, indicating the successful loading of these agents within the PVA_Lys polymeric nanofibers.

\subsection{Characterization of the membranes' mechanical properties}

The mechanical properties of the PVA_Lys, PVA_Lys_IBP, and PVA_Lys_LO membranes were determined in dry and wet conditions (Miguel et al., 2017). The Young's modulus, tensile strength, and elongation at break of the membranes and the values displayed by native skin are presented in Table 2. The Young's modulus obtained for 

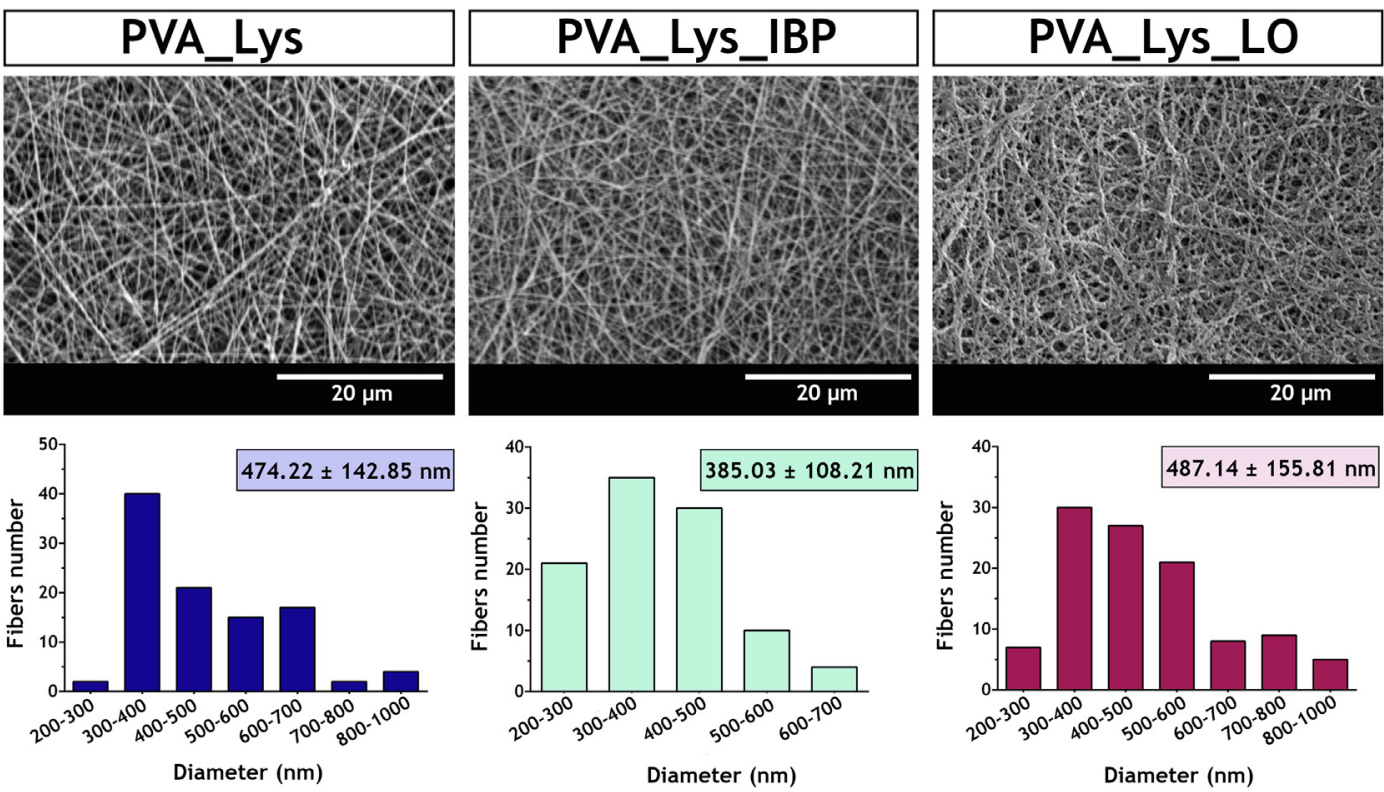

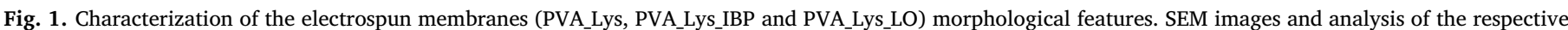
nanofibers' diameter distribution.

PVA_Lys, PVA_Lys_IBP, and PVA_Lys_LO membranes in the dry state were $\quad 74.67 \pm 10.00 \mathrm{MPa}, \quad 72.75 \pm 14.73 \mathrm{MPa}, \quad$ and $44.95 \pm 10.52 \mathrm{MPa}$, respectively. In wet conditions these values decreased to $1.44 \pm 0.55 \mathrm{MPa}, 2.10 \pm 0.15 \mathrm{MPa}$, and $0.89 \pm 0.01 \mathrm{MPa}$ for PVA_Lys, PVA_Lys_IBP, and PVA_Lys_LO membranes, respectively. Further, the PVA_Lys, PVA_Lys_IBP, and PVA_Lys_LO membranes showed values of Tensile Strength of $4.53 \pm 1.02 \mathrm{MPa}$, $2.78 \pm 0.66 \mathrm{MPa}$, and $2.89 \pm 1.94 \mathrm{MPa}$ in dry state, and $0.31 \pm 0.18 \mathrm{MPa}, 0.26 \pm 0.01 \mathrm{MPa}$ and $0.38 \pm 0.34 \mathrm{MPa}$ in wet conditions, respectively. On the other side, the PVA_Lys, PVA_Lys_IBP, and PVA_Lys_LO membranes can bear a strain of $8.18 \pm 5.23 \%$, $4.62 \pm 0.27 \%$, and $8.75 \pm 6.21 \%$ for the dry state and $19.23 \pm 5.13 \%, 14.95 \pm 1.45 \%$, and $17.00 \pm 4.13 \%$ in wet state, respectively.

The obtained results demonstrate that the drug incorporation methodologies did not impact on the membranes mechanical behaviour. In fact, all the tested groups presented similar mechanical properties (Young's modulus, tensile strength, and elongation at break). Further, in dry state, the mechanical parameters of the membranes are close to those exhibited by the native skin (Table 2). (Morgado et al., 2014) On the other side, the assays conducted under wet conditions reveal a decrease in both the Young modulus and tensile strength for all the tested samples. Such reduction on the membranes' mechanical performance under these conditions can be attributed to the highly
Table 2

Mechanical properties exhibited by the produced membranes. Data are presented as the mean \pm standard deviation $(n=5)$. The reference values for the native human skin were obtained from (Morgado et al., 2014).

\begin{tabular}{lllll}
\hline & & $\begin{array}{l}\text { Young Modulus } \\
\text { (MPa) }\end{array}$ & $\begin{array}{l}\text { Tensile strength } \\
\text { (MPa) }\end{array}$ & $\begin{array}{l}\text { Elongation at } \\
\text { break (\%) }\end{array}$ \\
\hline PVA_Lys & Dry & $74.67 \pm 10.00$ & $4.53 \pm 1.02$ & $8.18 \pm 5.23$ \\
& Wet & $1.44 \pm 0.55$ & $0.31 \pm 0.18$ & $19.23 \pm 5.13$ \\
PVA_Lys_IBP & Dry & $72.75 \pm 14.73$ & $2.78 \pm 0.66$ & $4.62 \pm 0.27$ \\
& Wet & $2.10 \pm 0.15$ & $0.26 \pm 0.01$ & $14.95 \pm 1.45$ \\
\multirow{2}{*}{ PVA_Lys_LO } & Dry & $44.95 \pm 10.52$ & $2.89 \pm 1.94$ & $8.75 \pm 6.21$ \\
& Wet & $0.89 \pm 0.01$ & $0.38 \pm 0.34$ & $17.00 \pm 4.13$ \\
\multirow{2}{*}{ Native skin } & - & $4.6-20$ & $5-30$ & $35-115$ \\
\hline
\end{tabular}

hydrophilic character of the membranes' raw materials (PVA and Lys) (Miao et al., 2017), that will prompt the interaction of membranes with the water molecules and consequently promote its swelling and decreasing the intermolecular forces established between the polymeric chains, leading to an increased molecular mobility (Sahputra et al., 2019). Similar results were also reported for CS/PEO electrospun membranes produced by An et al. (2009), and for CS electrospun membranes fabricated by Li et al. (2015).

Overall, the obtained results demonstrate the suitability of the produced membranes to provide adequate mechanical support during
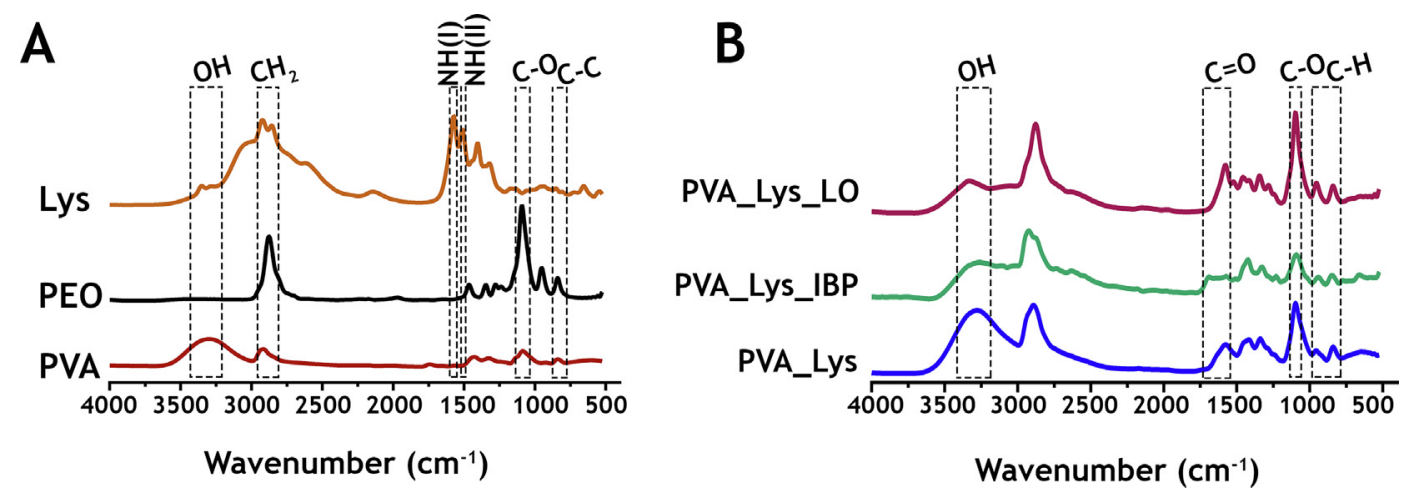

Fig. 2. ATR-FTIR analysis of the raw materials (A) and the produced electrospun membranes (B). 


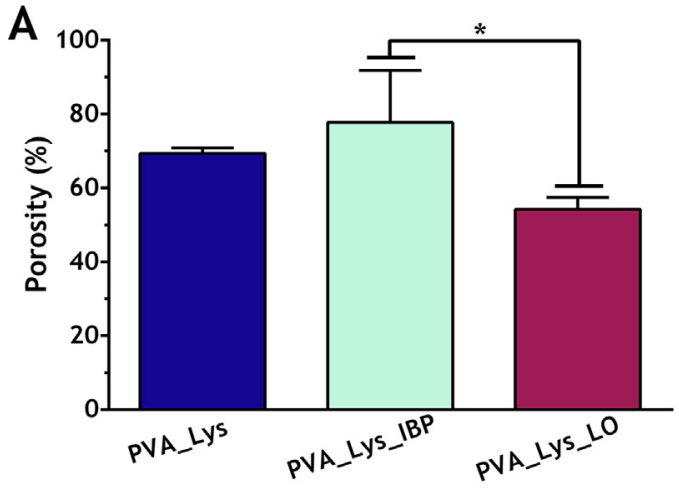

C

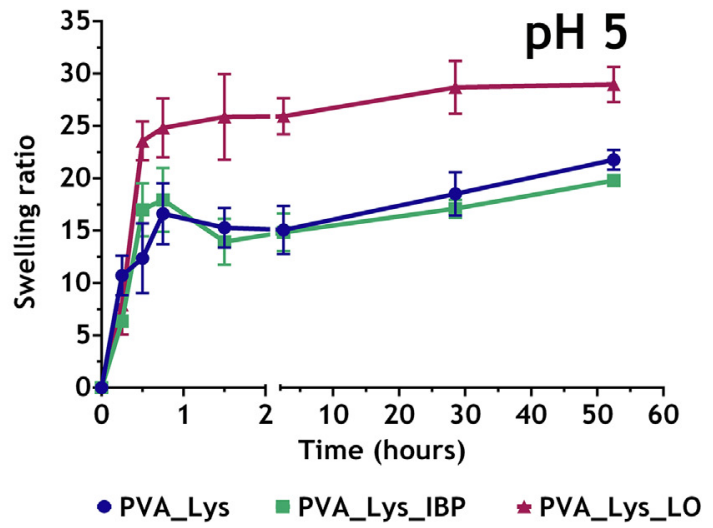

B

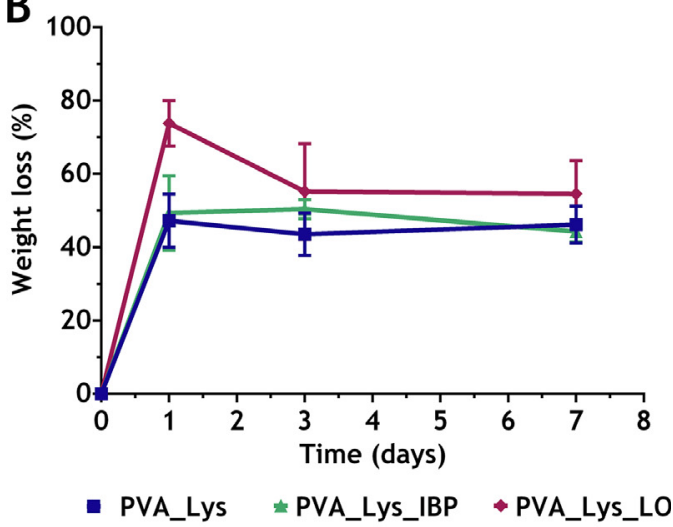

D

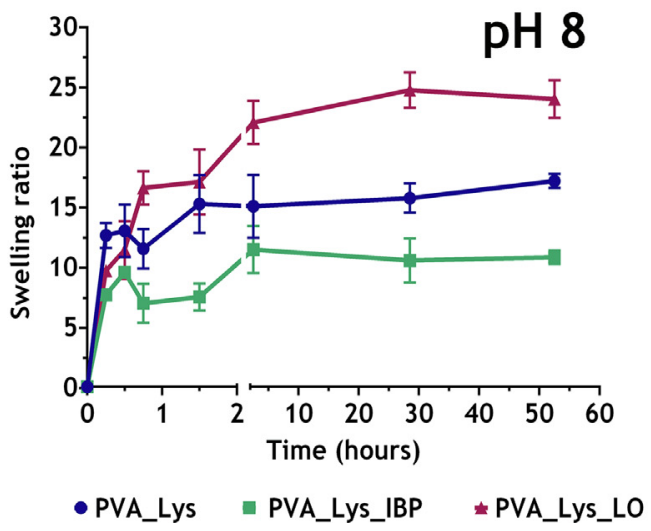

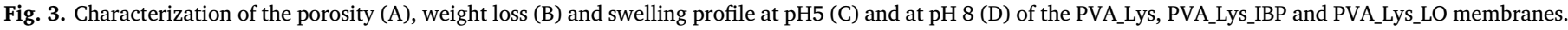
Data are presented as the mean \pm standard deviation, $n=5,{ }^{*} \mathrm{p}<0.05$ ).

the tissue remodelling process as well as the capacity to endure all the mechanical stresses that membranes suffer during its handling.

\subsection{Determination of the membranes' total porosity}

For skin regeneration purposes, the membranes' porosity determines their capacity to promote the exudate absorption, cell accommodation, distribution of nutrients and medium, gas exchanges, and waste excretion (Jin et al., 2013). According to the literature, a wound dressing must present an overall porosity within the range of $60-90 \%$ for an effective healing process to occur (Chong et al., 2007; Miguel et al., 2019b). Herein, the produced membranes presented a total porosity value of $69.29 \pm 1.54 \%, \quad 77.70 \pm 14.13 \%$ and $54.15 \pm 3.24 \%$ for PVA_Lys, PVA_Lys_IBP, and PVA_Lys_LO membranes, respectively (Fig. 3A). The obtained results revealed that the incorporation of IBP did not affect the total porosity of the membranes, whereas the LO adsorption at the nanofibers' surface promoted a decrease on their total porosity. Such differences are also corroborated with the structural alterations observed in the SEM images (Fig. 1). Indeed, the nanofibers of PVA_Lys_LO membranes exhibit fibers with higher mean diameters, increased surface roughness, and a lower number of void spaces. Such morphological alterations have been related with a decrease on the membranes porosity (Jabur et al., 2018). Nonetheless, all the produced membranes exhibited porosity values within the range considered as ideal to provide the required space for cellular accommodation, migration, nutrient exchange, and production of new ECM.

\subsection{Evaluation of the weight loss of the membranes}

Traditional wound dressings need to be removed from the wound site, in a process that can induce further lesions, increase the formation of scar tissue as well as facilitate the wound bacterial contamination (Jannesari et al., 2011). In this way, tissue engineering researchers have been focused on the development of biodegradable wound dressings that present a degradation profile compatible with the rate of new tissue formation. Herein, the weight loss of the different membranes was evaluated along 7 days of incubation (Fig. 3B). The results evidenced a more pronounced weight loss at day 1 for PVA_Lys_LO membranes $(73.79 \pm 6.18 \%)$ in comparison to the PVA_Lys $(47.20 \pm 7.27 \%)$ and PVA_Lys_IBP $(49.53 \pm 10.12 \%)$. Such difference can be attributed to the dissociation of the LO weak electrostatic interactions (occurring between the negatively charged acetate, aldehyde, and terpenoid groups of LO with the amine groups of Lys) with the surface of the PVA_Lys membrane and consequent drug release (Gudiksen et al., 2005; Jamróz et al., 2018; Yap et al., 2014).

On the other side, the weight loss profile exhibited after 3 and 7 days of incubation is similar for the different produced membranes. Such degradation profile of the membranes in a non-enzymatic solution is justified by the hydrolysis of the PVA and Lys when incubated at $37^{\circ} \mathrm{C}$ in PBS, simulating the in vivo conditions (Fountoulakis and Lahm, 1998; Santos et al., 2014).

\subsection{Characterization of electrospun membranes' swelling profile}

During the healing process, the majority of the exudate is produced along the inflammatory phase. The excessive accumulation of wound exudate can induce tissue maceration, and facilitate the occurrence of infections (Adderley, 2010). Therefore, the wound dressings must be able to avoid the excessive accumulation of exudates as well as maintain a moist environment at the wound site. Herein, the membranes capacity to adsorb fluids was characterized through the analysis of the 
A

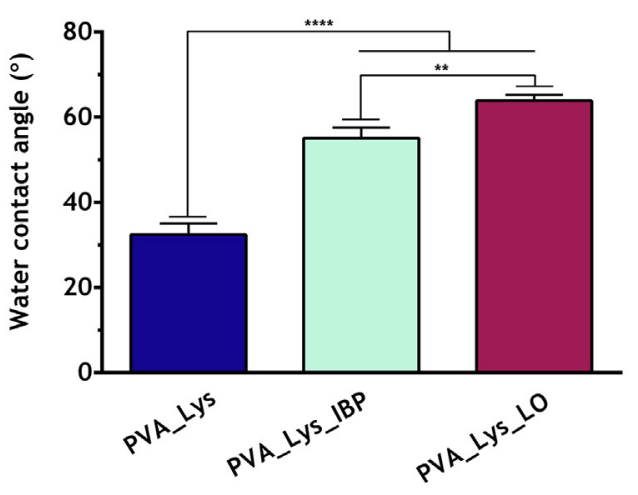

C

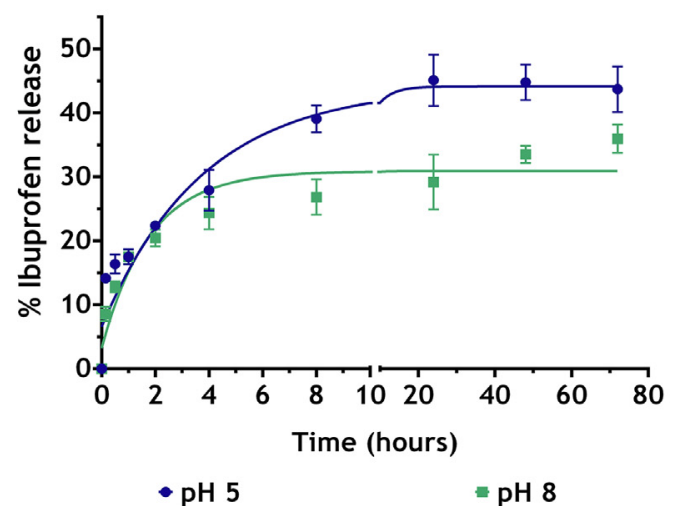

B

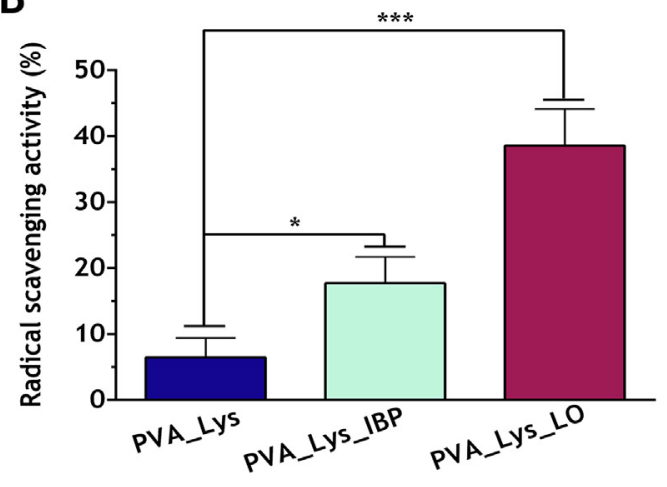

D

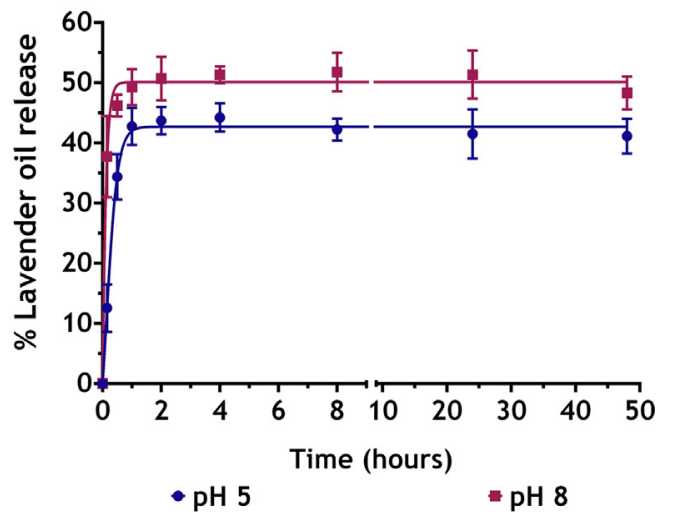

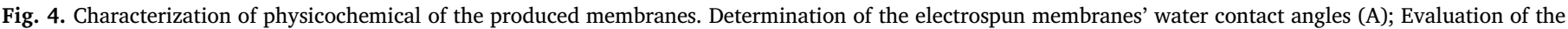

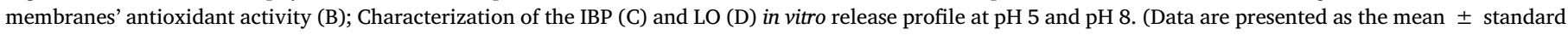
deviation, $\mathrm{n}=5$, *p $<0.05$, **p $<0.001$, *** $\mathrm{p}<0.0001$ and $* * * * \mathrm{p}<0.00001)$.

swelling profile. For that purpose, the membranes were incubated in PBS at pH 5 (Fig. 3C) and $\mathrm{pH} 8$ (Fig. 3D) to simulate the $\mathrm{pH}$ values of the native and injured skin, respectively (Miguel et al., 2019b; Morgado et al., 2014). The obtained results show that the membranes have the maximum water uptake capacity after $24 \mathrm{~h}$ of incubation, for both $\mathrm{pHs}$. However, it is quite notorious that the swelling ratio for PVA_Lys, PVA_Lys_IBP, and PVA_Lys_LO membranes was higher at $\mathrm{pH} 5(\approx 18.51$, $\approx 17.10, \approx 31.14)$ than at pH $8(\approx 15.69, \approx 10.51, \approx 24.68)$, respectively. The membranes swelling behaviour is explained by the hydrophilicity of its raw materials (PVA and Lys), as well as the protonation/ deprotonation of Lys functional groups associated with the $\mathrm{pH}$ variation. In fact, the Lys $\alpha$-amino groups will be deprotonated at $\mathrm{pH} 8$, yielding a neutral charge $(\approx 0)$ to the Lys molecule (Harms et al., 2008; Nolting et al., 2007). When the membrane is incubated at pH 5, the amino groups become protonated, leading to an increased electrostatic repulsion between them and, consequently, facilitating the establishment of stronger H-bonds with the water molecules (i.e. swelling occurs) (Jesus and Redinha, 2013). Considering all the gathered data, the swelling capacity exhibited by the membranes highlights their potential to remove the excess of exudate during at least $50 \mathrm{~h}$ of incubation, thus providing a suitable environment for the healing process to occur.

\subsection{Determination of water vapor transmission rate of membranes}

The wound dressings must also be permeable to moisture and gases in order to provide the optimal environment for the healing process (Xu et al., 2016). In this way, the determination of the membranes' WVTR revealed that the PVA_Lys membranes present a WVTR of $1660.75 \pm 171.58 \mathrm{~mL} / \mathrm{m}^{2} /$ day, whereas the PVA_Lys_IBP and PVA_Lys_LO membranes display a WVTR of $1572.50 \pm 151.74 \mathrm{~mL} / \mathrm{m}^{2} /$ day and $1397.37 \pm 220.44 \mathrm{~mL} / \mathrm{m}^{2} /$ day, respectively. These data show that the incorporation of both compounds (IBP and LO) into the PVA_Lys membrane promote a decrease on its WVTR values. However, these values are still superior to those presented by the majority of the commercially available wound dressings (e.g. $285 \pm 8 \mathrm{~g} / \mathrm{m}^{2} /$ day for Comfeel (Coloplast A/S); $394 \pm 12 \mathrm{~g} / \mathrm{m}^{2} /$ day for Bioclusive (JohnsonJohnson); $792 \pm 32 \mathrm{~g} / \mathrm{m}^{2} /$ day for Op Site (Smith \& Nephew; (Jonkman et al., 1988) and $120 \pm 30 \mathrm{~g} / \mathrm{m}^{2} /$ day for PVA-based asymmetric membranes (Morgado et al., 2014))). Furthermore, these results are in agreement with other previously reported for electrospun membranes (e.g. $1713 \pm 26 \mathrm{~mL} / \mathrm{m}^{2} /$ day for CS_Arginine membranes (Antunes et al., 2015), $1762.91 \pm 187.50 \mathrm{~mL} / \mathrm{m}^{2} /$ day for PCL_Hyaluronic acid/CS_zein bilayer membrane (Figueira et al., 2016), and $1353 \mathrm{~g} / \mathrm{m}^{2} /$ day for CS electrospun membranes (Naseri et al., 2014)).

\subsection{Characterization of membranes' wettability through the measurement of water contact angles}

The determination of the water contact angle (WCA) at the surface of the electrospun membranes provide important insights about the host tissue interaction with the material surface. According to the literature, a hydrophilic surface displays a WCA $<90^{\circ}$, a moderate hydrophilic surface present $40^{\circ}<$ WCA $<70^{\circ}$, while a hydrophobic substrate possesses a WCA $>90^{\circ}$ (Arima and Iwata, 2007; Oliveira et al., 2014). Herein, the PVA_Lys, PVA_Lys_IBP, and PVA_Lys_LO membranes exhibited a WCA of $32.38 \pm 2.69^{\circ}, 55.05 \pm 2.55^{\circ}$ and $63.83 \pm 1.43^{\circ}$, respectively (as can be observed in Fig. 4A). The PVA_Lys membrane presents the lower WCA, revealing a higher hydrophilic character. Such feature is justified by the presence of PVA and Lys in the composition of the membrane, which are both composed of 
A

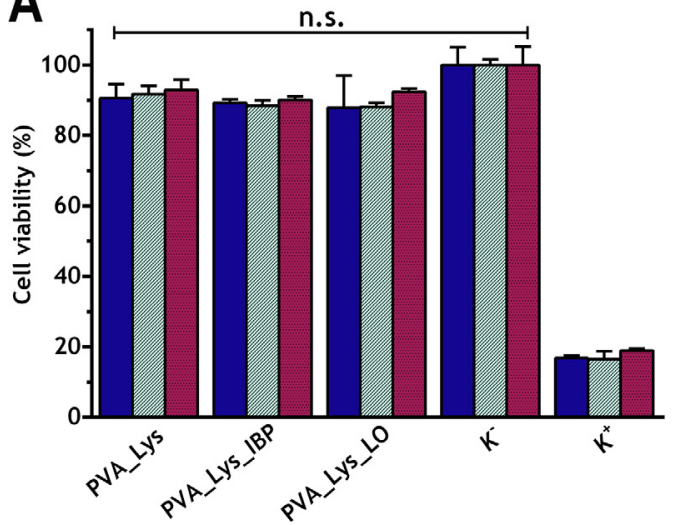

Day 1
Day 3
Day 7
B

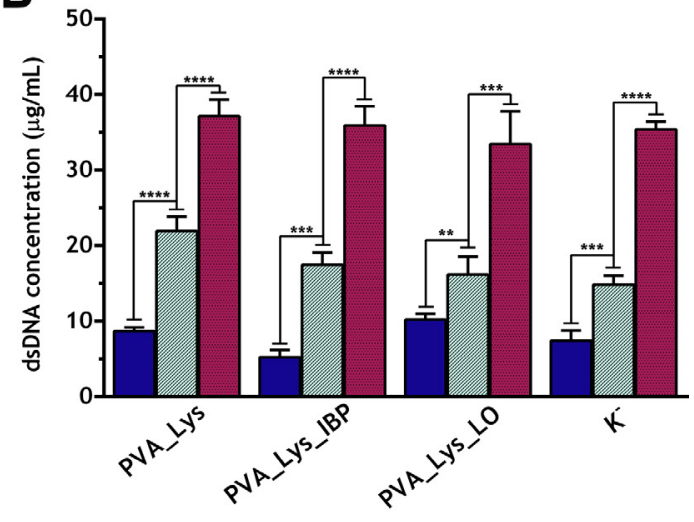

Day 1 Day 3

$\square$ Day 7

\section{C}
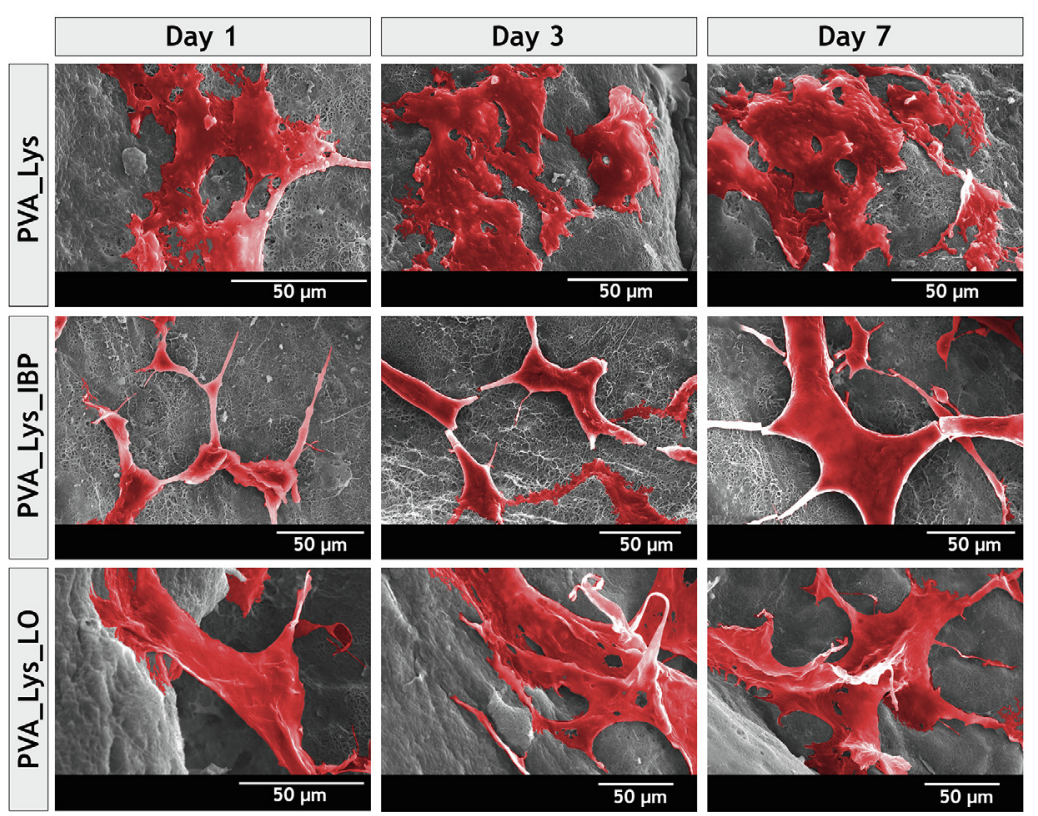

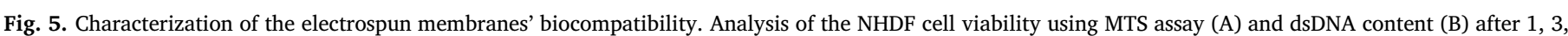

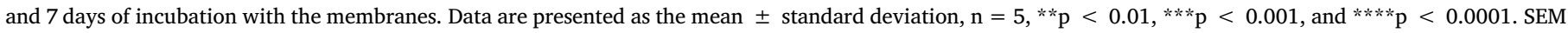
images of NHDF cells seeded at the surface of the electrospun membranes after 1, 3, and 7 days (C).

highly hydrophilic groups (Miao et al., 2017). On the other side, the IBP and LO incorporation prompted an increase on the WCA, resulting from the hydrophobic character of the incorporated agents (Balasubramanian and Kodam, 2014; Lowe et al., 1999). However, the WCA values obtained for these two groups are comprehended between $40^{\circ}$ and $70^{\circ}$, corresponding to a moderate hydrophilic character, which is considered to be suitable for encouraging the cell attachment and proliferation at the membranes' surface (Miguel et al., 2017; Oliveira et al., 2014).

\subsection{Evaluation of the antioxidant activity of the membranes}

There is a well-known relation between the inflammatory phase of the wound healing process and the exuberant production of reactive oxygen species (ROS). Imbalances on ROS production and their elimination may originate a condition of oxidative stress, which is commonly observed in chronic inflammatory diseases (Pérez et al., 2017). Thus, the ROS can trigger the pro-inflammatory cytokines secretion and the activity of matrix metalloproteases, which degrade the ECM and consequently impair the healing process.

In this work, the electrospun membranes were incubated with a solution containing DPPH radicals and their radical scavenging activity was evaluated (Fig. 4B). The PVA_Lys exhibit a radical scavenging activity of $6.42 \pm 2.94 \%$, whereas the PVA_Lys_IBP and PVA_Lys_LO present higher radical scavenging values, $17.68 \pm 3.99 \%$ and $38.54 \pm 5.58 \%$, respectively. The IBP scavenging activity is attributed to the phenyl group and its reactivity with the ROS (Costa et al., 2006). Further, in in vivo conditions the IBP also decreases the ROS formation by inhibiting the COXs (Huang et al., 2005; Van Antwerpen and Neve, 2004). In turn, the membranes containing the LO were the ones that showed the highest antioxidant activity. The LO is an essential oil which has been widely applied in food processing due to its antioxidant activity, which is crucial to confer protection to food products against oxidative rancidity (Danh et al., 2012; Yang et al., 2010). The LO' antioxidant activity arises from the presence of phenolic compounds in its composition, which act as free radical acceptors that delay/inhibit the autoxidation initiation step or prevents its propagation (Eça et al., 2014; Kacániová et al., 2012). 


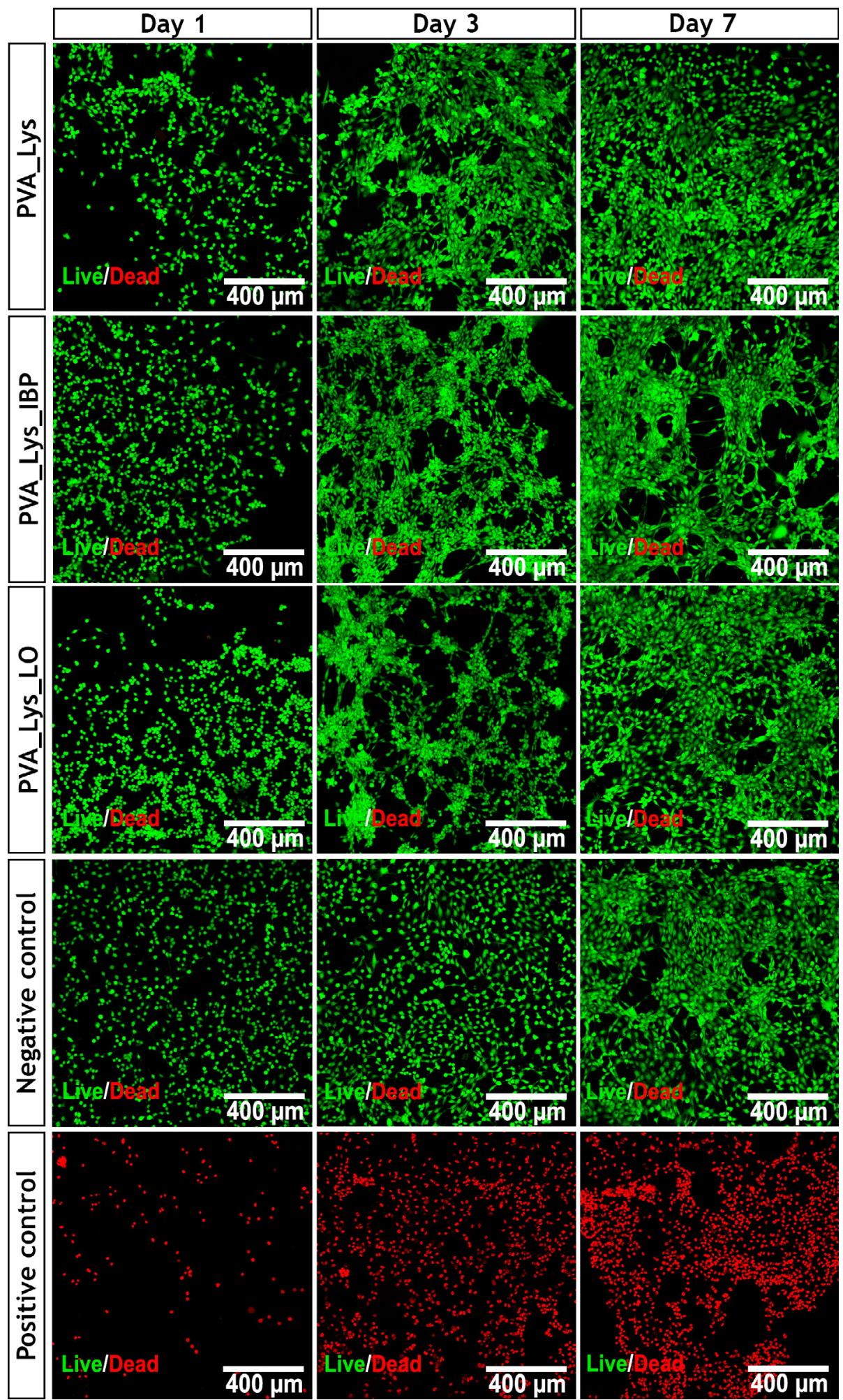

Fig. 6. Fluorescence microscopic images of a Live/Dead assay: NHDFs cultured on electrospun membranes' surface after, 1,3 and 7 days. Green channel: viable cells labelled with Calcein; red channel: dead cells stained with Propidium iodide. (For interpretation of the references to color in this figure legend, the reader is referred to the web version of this article.)

\subsection{Characterization of drug release profile of IBP and LO from nanofibers}

In this study, IBP and LO were selected to be incorporated into PVA_Lys electrospun membranes, for providing anti-inflammatory, antibacterial or antioxidant properties. The EE and LE values obtained for IBP were $84.30 \pm 5.81 \%$ and $71.77 \pm 7.77 \%$, whereas for LO were
$83.42 \pm 2.69 \%$ and $68.63 \pm 9.77 \%$, respectively. Such results revealed that both IBP and LO can be efficiently incorporated into PVA_Lys nanofibrous membrane through the electrospinning technique using the blend electrospinning and surface physical adsorption methodologies, respectively.

Then, the IBP and LO release profiles from PVA_Lys nanofibrous 

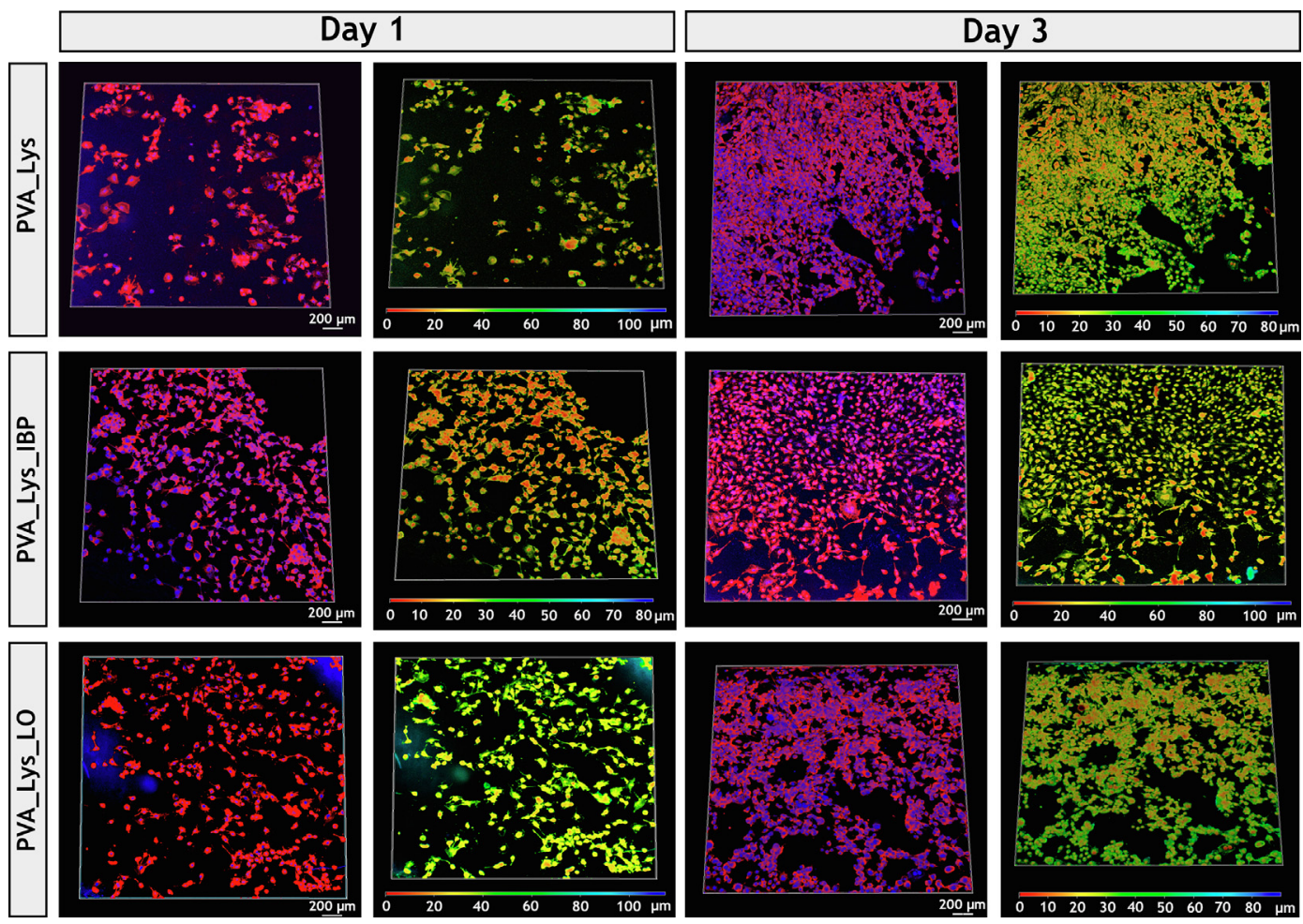

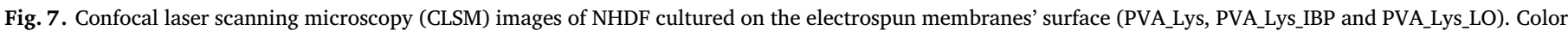

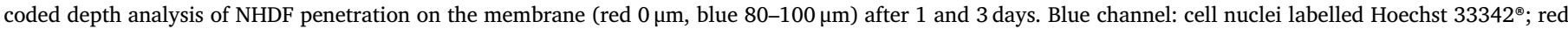

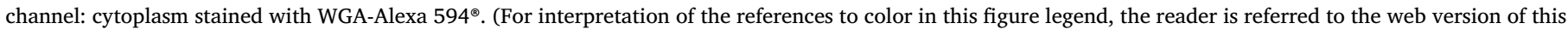
article.)

membrane were characterized at $\mathrm{pH} 5$ (found in native skin) and $\mathrm{pH} 8$ (injured skin). In relation to IBP (Fig. 4C), the results show that the IBP release from the nanofibers, at both $\mathrm{pHs}$, occurred in a gradual manner reaching its maximum after $24 \mathrm{~h}$ of incubation, $45.09 \pm 4.02 \%$ and $29.17 \pm 4.29 \%$ of IBP were released at $\mathrm{pH} 5$ and $\mathrm{pH} 8$, respectively. The impact of the $\mathrm{pH}$ on the IBP release profile is in accordance with the higher swelling exhibited by the membranes at $\mathrm{pH} 5$, which facilitate the drug diffusion from the nanofiber polymeric matrix to the external media.

On the other side, the $\mathrm{LO}$ release profile (Fig. 4D) is characterized by an initial burst release that occurs in the first $4 \mathrm{~h}$ of immersion in PBS $(44.21 \pm 2.33 \%$ and $51.32 \pm 1.39 \%$ at $\mathrm{pH} 5$ and $\mathrm{pH} 8$, respectively), followed by a stabilization of the drug release until $48 \mathrm{~h}$. The initial LO burst release is justified by the method used to perform its incorporation into PVA_Lys membrane. In fact, the surface physical adsorption method used just promotes the LO immobilization at the surface of the polymeric matrix through electrostatic interactions (positively charged amine groups of Lys and negatively charged terpene groups available on LO structure) (Balasubramanian and Kodam, 2014; Gudiksen et al., 2005; Jamróz et al., 2018; Yap et al., 2014). Therefore, the LO will be immediately released from the nanofibers' surface, which is crucial to prevent the bacterial infections that usually occur within the first hours after the wound dressing application (Negut et al., 2018).

\subsection{Characterization of biological properties of electrospun membranes}

The biological properties of the electrospun membranes were evaluated using the NHDF as model cells. These cells were selected due to their pivotal role in the wound healing process (Barrientos et al., 2008). They are responsible for the production of collagen, growth factors, adhesive molecules, and cytokines that play active roles during the reestablishment of the injured skin (Barrientos et al., 2008). The optical microscopic images of NHDF cells seeded in contact with electrospun membranes were acquired after 1, 3 and 7 days of incubation (Fig. S3). The images show that the cell morphology was not affected by the presence of PVA_Lys membranes, since they exhibit an elongated morphology quite similar to cells found in the negative control (i.e. cells incubated just with culture medium).

Additionally, the membranes' cytotoxic profile was analysed through the MTS assay over 1, 3, and 7 days (Fig. 5A). The obtained data show that all the produced membranes did not induce any cytotoxic effect on NHDFs, over the 7 days. Further, the concentration of IBP $(1 \mathrm{mg} / \mathrm{mL})$ and LO $(1.3 \mathrm{mg} / \mathrm{mL})$ incorporated into the nanofibers did not compromise fibroblasts' viability. Likewise, membranes' biocompatibility was also corroborated by the dsDNA assay, where the results show that no significant differences were noticed in dsDNA content of cells in contact with membranes or just in the presence of culture medium over a period of 7 days (Fig. 5B).

Moreover, the SEM images (Fig. 5C) highlight the bioadhesive properties of the electrospun membranes, showing that the cells adhered and spread at the surface of the membranes, along 7 days of incubation. Such occur essentially due to the presence of positively charged amine groups on Lys backbone (in membrane' composition), which interact with negatively charged glycosaminoglycans found in the cell membrane (Kim et al., 2011). In fact, the Lys and their derivatives have been used for coating the cell culture dishes' surface due to their biocompatibility, capacity to enhance cell adhesion, and relatively good solubility in water (Wang et al., 2015).

Furthermore, the Live/Dead assays allow to characterize the cell survival at the membranes' surface, by simultaneously staining both live (green labelled) and dead (red labelled) cells with Calcein and Propidium iodide, respectively. The confocal microscopy images (Fig. 6) demonstrated that the fibroblasts remain viable and their number increase along time, corroborating the results obtained in the MTS and dsDNA assays.

In addition, the cell proliferation, attachment and migration into the 
A

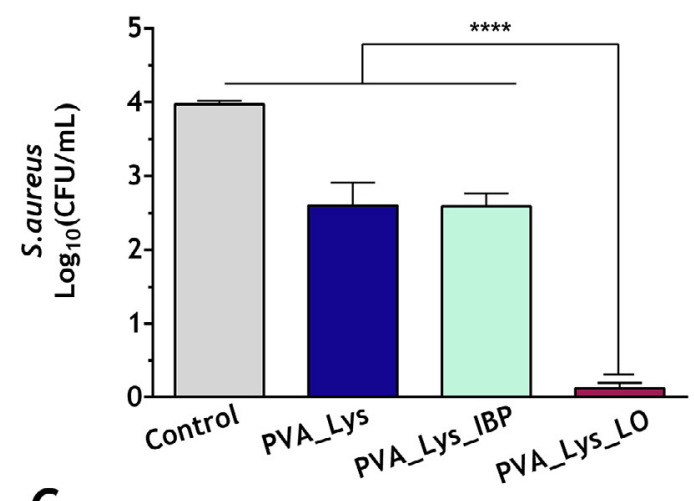

C

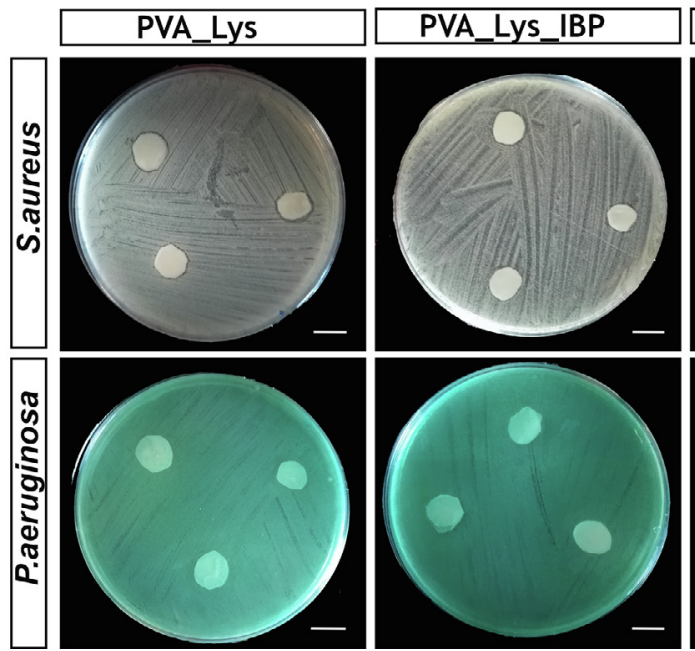

B
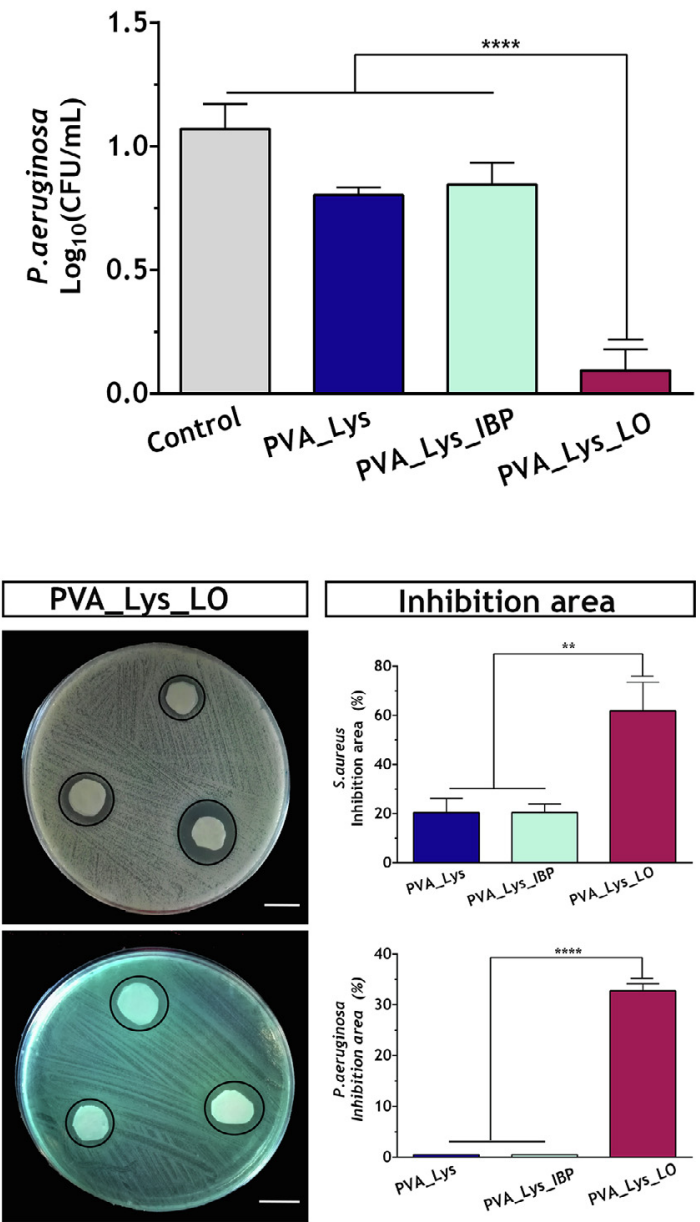

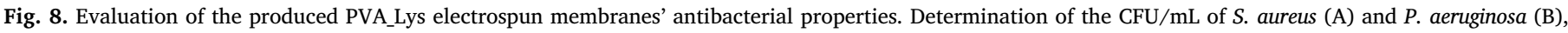

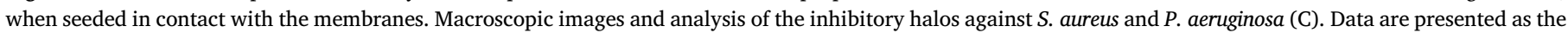
mean \pm standard deviation, $\mathrm{n}=5,{ }^{* *} \mathrm{p}<0.001$, **** $\mathrm{p}<0.0001$.

membranes were also characterized through CLSM analysis (Fig. 7). The results revealed that the fibroblast cells maintained a normal phenotype and remained biologically active at the surface of electrospun membranes for 3 days, corroborating the data obtained through SEM analysis. Further, the color-coded depth analysis of the membranes indicated that fibroblasts migrated within the electrospun membranes, with cells localized between 30 and $60 \mu \mathrm{m}$ of depth within the membranes' polymeric matrix. Overall, the obtained data clearly demonstrate the excellent biocompatibility of the PVA_Lys electrospun membranes based-drug delivery systems, encouraging their application as a wound dressing.

\subsection{Evaluation of antimicrobial properties of electrospun membranes}

An open wound provides a favourable niche for microbial colonization, which will impair the healing process. Additionally, the microbial infections are one of the main causes of the high rates of morbidity and mortality found in the wound management area. So, the development of wound dressings displaying antimicrobial activity is crucial to avoid bacterial penetration and infections. Herein, the membranes' antimicrobial properties were evaluated against $S$. aureus (Gram-positive bacteria) and $P$. aeruginosa (Gram-negative bacteria), which are the most common microorganisms found in skin infections (Simões et al., 2018b).

The results show that PVA_Lys membranes can impair or even inhibit bacterial growth. The PVA_Lys, PVA_Lys_IBP, and PVA_Lys_LO induced a bacterial growth inhibition of $34.55 \pm 7.87 \%$, $34.85 \pm 4.48 \%$, and $96.96 \pm 1.84 \%$ for $S$. aureus, and $25.00 \pm 2.90 \%, 21.00 \pm 8.23 \%$, and $91.18 \pm 7.88 \%$ for $P$. aeruginosa, respectively (as can be observed in Fig. 8A and B). Similar results were observed in the agar diffusion assay, with the PVA_Lys, PVA_LyS_IBP and PVA_Lys_LO membranes (Fig. 8C) presenting an inhibitory area of $20.43 \pm 5.78 \%, 20.49 \pm 3.42 \%$ and $61.76 \pm 11.75 \%$ against S. aureus, and $0.51 \pm 0.01 \%, 0.45 \pm 0.02 \%$ and $32.77 \pm 1.36 \%$ for $P$. aeruginosa, respectively. Additionally, SEM images (Fig. S4) also showed that no biofilm formation occurred at PVA_Lys, PVA_Lys_IBP and PVA_Lys_LO membranes' surface when samples were incubated with $S$. aureus. On the other hand, when membranes were incubated with $P$. aeruginosa, biofilm formation was only inhibited at the surface of PVA_Lys_LO membrane.

These results are in agreement with the data available in the literature for LO (Cavanagh and Wilkinson, 2002; Lis-Balchin, 2003). The LO's antibacterial activity is justified by the presence of terpene compounds (e.g. linalool, linalyl acetate, 1,8-cineole, $\beta$-ocimene, terpinen-4ol). Furthermore, the electronegative compounds present on LO's composition will also interfere on the bacteria biological processes (e.g. electron transfer, protein translocation, phosphorylation steps) and therefore, inhibit the growth of the microorganism (Balasubramanian and Kodam, 2014; Yap et al., 2014). On the other hand, the essential oils can also disrupt the bacterial cell wall and cytoplasmic membrane 
inducing the cell lysis and subsequent leakage of intracellular compounds (Lopez-Romero et al., 2015; Sakkas et al., 2018). However, in this work, the antibacterial activity of LO was influenced by the microorganisms' structure, i.e., the PVA_Lys_LO presented an enhanced antibacterial effect against $S$. aureus. Such result may be linked to the composition of the cell wall on gram-negative bacteria, which is composed mainly of lipopolysaccharides limiting the diffusion of hydrophobic compounds, such as the essential oils (Chouhan et al., 2017; Miguel et al., 2019b). On the other side, the antibacterial activity of PVA_Lys and PVA_Lys_IBP membranes against $S$. aureus can be attributed to the interaction of the positively charged amine groups of Lys with the negatively charged groups available at the bacteria surface, which will lead to an increased cell wall permeability and consequently the leakage of intracellular compounds (Hyldgaard et al., 2014).

\section{Conclusions}

In this work, nanofibrous membranes composed of PVA and Lys were produced using the electrospinning technique. Blend electrospinning was used to incorporate IBP into the membranes in order to control the inflammatory phase (1-3 days after skin injury). On the other hand, LO was loaded into the membranes through surface physical adsorption. Such procedure leads to an initial burst release of LO, that provides an initial aseptic environment at the wound site, which is crucial for avoiding the bacterial colonization. On the other side, all the electrospun membranes presented suitable morphological, mechanical, physicochemical and biological properties to be used as wound dressings. Further, the LO incorporation on PVA_Lys membranes mediated a strong antibacterial effect against both $S$. aureus and $P$. aeruginosa.

In the near future, the in vitro and in vivo anti-inflammatory properties of IBP will be further evaluated. Moreover, the synergic effect of the IBP and LO incorporation into the same electrospun membrane will be further characterized to support the application of these membranes as multifunctional wound dressings, that can deliver anti-inflammatory and antibacterial compounds in a time frame that match the specific requirements of the healing process. Additionally, an alternative LO incorporation method may be hypothesized (e.g. chemical immobilization of LO at membranes' surface) to achieve a more sustained and prolonged drug release profile.

\section{Declaration of Competing Interest}

The authors declare that they have no known competing financial interests or personal relationships that could have appeared to influence the work reported in this paper.

\section{Acknowledgments}

The authors would like to acknowledge Abílio Silva for help in the mechanical assays. Financial support was provided by FEDER funds through the POCI -COMPETE 2020 -Operational Programme Competitiveness and Internationalization in Axis I -Strengthening research, technological development, and innovation (Project POCI-010145- FEDER-007491) and National Funds by FCT -Foundation for Science and Technology (Project UID/Multi/00709/2013). The funding from CENTRO-01-0145-FEDER-028989 and POCI-01-0145-FEDER031462 is also acknowledged. Sónia P. Miguel and André F. Moreira acknowledge their Ph.D. fellowships from FCT (SFRH/BD/109563/ 2015 and SFRH/BD/109482/2015, respectively).

\section{Appendix A. Supplementary data}

Supplementary data to this article can be found online at https:// doi.org/10.1016/j.ijpharm.2019.118640.

\section{References}

Adderley, U.J., 2010. Managing wound exudate and promoting healing. Br. J. Commun. Nurs. 15, S15-S20.

Akduman, C., Özgüney, I., Kumbasar, E.P.A., 2016. Preparation and characterization of naproxen-loaded electrospun thermoplastic polyurethane nanofibers as a drug delivery system. Mater. Sci. Eng. C 64, 383-390.

Amariei, G., Kokol, V., Vivod, V., Boltes, K., Letón, P., Rosal, R., 2018. Biocompatible antimicrobial electrospun nanofibers functionalized with $\varepsilon$-poly-l-lysine. Int. J. Pharm. 553, 141-148.

An, J., Zhang, H., Zhang, J., Zhao, Y., Yuan, X., 2009. Preparation and antibacterial activity of electrospun chitosan/poly (ethylene oxide) membranes containing silver nanoparticles. Colloid Polym. Sci. 287, 1425-1434.

Antunes, B.P., Moreira, A.F., Gaspar, V., Correia, I., 2015. Chitosan/arginine-chitosan polymer blends for assembly of nanofibrous membranes for wound regeneration. Carbohydr. Polym. 130, 104-112.

Arima, Y., Iwata, H., 2007. Effect of wettability and surface functional groups on protein adsorption and cell adhesion using well-defined mixed self-assembled monolayers. Biomaterials 28, 3074-3082.

Balasubramanian, K., Kodam, K.M., 2014. Encapsulation of therapeutic lavender oil in an electrolyte assisted polyacrylonitrile nanofibres for antibacterial applications. RSC Adv. 4, 54892-54901.

Barrientos, S., Stojadinovic, O., Golinko, M.S., Brem, H., Tomic-Canic, M., 2008. Growth factors and cytokines in wound healing. Wound Repair Regener. 16, 585-601.

Braghirolli, D.I., Steffens, D., Pranke, P., 2014. Electrospinning for regenerative medicine: a review of the main topics. Drug Discovery Today 19, 743-753.

Cabral, C.S., Miguel, S.P., de Melo-Diogo, D., Louro, R.O., Correia, I.J., 2019. Green reduced graphene oxide functionalized 3D printed scaffolds for bone tissue regeneration. Carbon 146, 513-523.

Carreras, N., Acuña, V., Martí, M., Lis, M., 2013. Drug release system of ibuprofen in PCLmicrospheres. Colloid Polym. Sci. 291, 157-165.

Cavanagh, H., Wilkinson, J., 2002. Biological activities of lavender essential oil. Phytother. Res. 16, 301-308.

Chong, E., Phan, T., Lim, I., Zhang, Y., Bay, B., Ramakrishna, S., Lim, C., 2007. Evaluation of electrospun PCL/gelatin nanofibrous scaffold for wound healing and layered dermal reconstitution. Acta Biomater. 3, 321-330.

Chou, S.-F., Carson, D., Woodrow, K.A., 2015. Current strategies for sustaining drug release from electrospun nanofibers. J. Control. Release 220, 584-591.

Chouhan, S., Sharma, K., Guleria, S., 2017. Antimicrobial activity of some essential oils-present status and future perspectives. Medicines 4, 58.

Costa, D., Moutinho, L., Lima, J.L.F.C., Fernandes, E., 2006. Antioxidant activity and inhibition of human neutrophil oxidative burst mediated by arylpropionic acid nonsteroidal anti-inflammatory drugs. Biol. Pharm. Bull. 29, 1659-1670.

Danh, L.T., Triet, N.D.A., Zhao, J., Mammucari, R., Foster, N., 2012. Antioxidant activity, yield and chemical composition of lavender essential oil extracted by supercritical CO2. J. Supercrit. Fluids 70, 27-34.

Eça, K.S., Sartori, T., Menegalli, F.C., 2014. Films and edible coatings containing antioxidants-a review. Br. J. Food Technol. 17, 98-112.

Figueira, D.R., Miguel, S.P., de Sá, K.D., Correia, I.J., 2016. Production and characterization of polycaprolactone-hyaluronic acid/chitosan-zein electrospun bilayer nanofibrous membrane for tissue regeneration. Int. J. Biol. Macromol. 93, 1100-1110.

Fountoulakis, M., Lahm, H.-W., 1998. Hydrolysis and amino acid composition analysis of proteins. J. Chromatogr. A 826, 109-134.

Gizaw, M., Thompson, J., Faglie, A., Lee, S.-Y., Neuenschwander, P., Chou, S.-F., 2018. Electrospun fibers as a dressing material for drug and biological agent delivery in wound healing applications. Bioengineering 5, 9.

Goyal, R., Macri, L.K., Kaplan, H.M., Kohn, J., 2016. Nanoparticles and nanofibers for topical drug delivery. J. Control. Release 240, 77-92.

Gudiksen, K.L., Gitlin, I., Yang, J., Urbach, A.R., Moustakas, D.T., Whitesides, G.M., 2005. Eliminating positively charged lysine E-NH3 groups on the surface of carbonic anhydrase has no significant influence on its folding from sodium dodecyl sulfate. J. Am. Chem. Soc. 127, 4707-4714.

Guo, S.A., Di Pietro, L.A., 2010. Factors affecting wound healing. J. Dent. Res. 89, 219-229.

Gurtner, G.C., Werner, S., Barrandon, Y., Longaker, M.T., 2008. Wound repair and regeneration. Nature 453, 314.

Harms, M.J., Schlessman, J.L., Chimenti, M.S., Sue, G.R., Damjanović, A., García-Moreno, E., B, 2008. A buried lysine that titrates with a normal pKa: role of conformational flexibility at the protein-water interface as a determinant of pKa values. Protein Sci. $17,833-845$.

Hart, J., 2002. Inflammation 1: its role in the healing of acute wounds. J. Wound Care 11, 205-209.

Huang, D., Ou, B., Prior, R.L., 2005. The chemistry behind antioxidant capacity assays. J. Agric. Food. Chem. 53, 1841-1856.

Hyldgaard, M., Mygind, T., Vad, B.S., Stenvang, M., Otzen, D.E., Meyer, R.L., 2014. The antimicrobial mechanism of action of epsilon-poly-l-lysine. Appl. Environ. Microbiol. 80, 7758-7770.

Im, J.S., Yun, J., Lim, Y.-M., Kim, H.-I., Lee, Y.-S., 2010. Fluorination of electrospun hydrogel fibers for a controlled release drug delivery system. Acta Biomater. 6 , 102-109.

Jabur, A., Najim, M., Al-Rahman, S.A.A., 2018. Study the effect of flow rate on some physical properties of different polymeric solutions. IOP Publishing. J. Phys.: Conf. Seri., 012069.

Jamróz, E., Juszczak, L., Kucharek, M., 2018. Investigation of the physical properties, antioxidant and antimicrobial activity of ternary potato starch-furcellaran-gelatin films incorporated with lavender essential oil. Int. J. Biol. Macromol. 114, 1094-1101.

Jannesari, M., Varshosaz, J., Morshed, M., Zamani, M., 2011. Composite poly (vinyl alcohol)/poly (vinyl acetate) electrospun nanofibrous mats as a novel wound dressing matrix for controlled release of drugs. Int. J. Nanomed. 6, 993. 
Jatoi, A.W., Ogasawara, H., Kim, I.S., Ni, Q.-Q., 2019. Polyvinyl alcohol nanofiber based three phase wound dressings for sustained wound healing applications. Mater. Lett. $241,168-171$.

Jesus, A.L., Redinha, J., 2013. Molecular insight into the amine-water interaction: a combined vibrational, energetic and NBO/NEDA study. Comput. Theor. Chem. 1023, 74-82.

Jin, G., Prabhakaran, M.P., Kai, D., Annamalai, S.K., Arunachalam, K.D., Ramakrishna, S., 2013. Tissue engineered plant extracts as nanofibrous wound dressing. Biomaterials 34, 724-734.

Jonkman, M.F., Molenaar, I., Nieuwenhuis, P., Bruin, P., Pennings, A.J., 1988. New method to assess the water vapour permeance of wound coverings. Biomaterials 9 , 263-267.

Kacániová, M., Vukovic, N., Hleba, L., Bobková, A., Pavelková, A., Rovná, K., Arpásová, H., 2012. Antimicrobial and antiradicals activity of Origanum vulgare L. and Thymus vulgaris essential oils. J. Microbiol. Biotechnol. Food Sci. 2, 263.

Kim, Y.H., Baek, N.S., Han, Y.H., Chung, M.-A., Jung, S.-D., 2011. Enhancement of neuronal cell adhesion by covalent binding of poly-d-lysine. J. Neurosci. Meth. 202, 38-44.

Li, Q., Wang, X., Lou, X., Yuan, H., Tu, H., Li, B., Zhang, Y., 2015. Genipin-crosslinked electrospun chitosan nanofibers: determination of crosslinking conditions and evaluation of cytocompatibility. Carbohydr. Polym. 130, 166-174.

Lis-Balchin, M., 2003. Lavender: The Genus Lavandula. CRC Press.

Liu, S., Hu, C., Li, F., Li, X.-J., Cui, W., Fan, C., 2012. Prevention of peritendinous adhesions with electrospun ibuprofen-loaded poly (L-lactic acid)-polyethylene glycol fibrous membranes. Tissue Eng. Part A 19, 529-537.

Lopez-Romero, J.C., González-Ríos, H., Borges, A., Simões, M., 2015. Antibacterial effects and mode of action of selected essential oils components against Escherichia coli and Staphylococcus aureus. Evidence-Based Complementary and Alternative Medicine 2015.

Lowe, T.L., Tenhu, H., Tylli, H., 1999. Effect of hydrophobicity of a drug on its release from hydrogels with different topological structures. J. Appl. Polym. Sci. 73, 1031-1039.

Mahanta, N., Valiyaveettil, S., 2011. Surface modified electrospun poly (vinyl alcohol) membranes for extracting nanoparticles from water. Nanoscale 3, 4625-4631.

Majd, S.A., Khorasgani, M.R., Moshtaghian, S.J., Talebi, A., Khezri, M., 2016. Application of Chitosan/PVA Nano fiber as a potential wound dressing for streptozotocin-induced diabetic rats. Int. J. Biol. Macromol. 92, 1162-1168.

Mekhail, M., Jahan, K., Tabrizian, M., 2014. Genipin-crosslinked chitosan/poly-1-lysine gels promote fibroblast adhesion and proliferation. Carbohydr. Polym. 108, 91-98.

Miao, Y., Yang, R., Deng, D.Y., Zhang, L.-M., 2017. Poly (L-lysine) modified zein nanofibrous membranes as efficient scaffold for adhesion, proliferation, and differentiation of neural stem cells. RSC Adv. 7, 17711-17719.

Miguel, S., Ribeiro, M., Coutinho, P., Correia, I., 2017. Electrospun Polycaprolactone/aloe Vera_Chitosan Nanofibrous asymmetric membranes aimed for wound healing applications. Polymers 9, 183.

Miguel, S.P., Figueira, D.R., Simões, D., Ribeiro, M.P., Coutinho, P., Ferreira, P., Correia, I.J., 2018. Electrospun polymeric nanofibres as wound dressings: a review. Colloids Surf., B 169, 60-71.

Miguel, S.P., Sequeira, R.S., Moreira, A.F., Cabral, C.S., Mendonça, A.G., Ferreira, P., Correia, I.J., 2019a. An overview of electrospun membranes loaded with bioactive molecules for improving the wound healing process. Eur. J. Pharm. Biopharm. 139, $1-22$.

Miguel, S.P., Simões, D., Moreira, A.F., Sequeira, R.S., Correia, I.J., 2019b. Production and characterization of electrospun silk fibroin based asymmetric membranes for wound dressing applications. Int. J. Biol. Macromol. 121, 524-535.

Mohiti-Asli, M., Saha, S., Murphy, S., Gracz, H., Pourdeyhimi, B., Atala, A., Loboa, E., 2017. Ibuprofen loaded PLA nanofibrous scaffolds increase proliferation of human skin cells in vitro and promote healing of full thickness incision wounds in vivo. J. Biomed. Mater. Res. B Appl. Biomater. 105, 327-339.

Mohseni, M., Shamloo, A., Aghababaie, Z., Afjoul, H., Abdi, S., Moravvej, H., Vossoughi, M., 2019. A comparative study of wound dressings loaded with silver sulfadiazine and silver nanoparticles: in vitro and in vivo evaluation. Int. J. Pharm. 564, 350-358.

Monteiro, N., Martins, M., Martins, A., Fonseca, N.A., Moreira, J.N., Reis, R.L., Neves, N.M., 2015. Antibacterial activity of chitosan nanofiber meshes with liposomes im mobilized releasing gentamicin. Acta Biomater. 18, 196-205.

Moreira, A.F., Gaspar, V.M., Costa, E.C., de Melo-Diogo, D., Machado, P., Paquete, C.M., Correia, I.J., 2014. Preparation of end-capped pH-sensitive mesoporous silica nanocarriers for on-demand drug delivery. Eur. J. Pharm. Biopharm. 88, 1012-1025.

Morgado, P.I., Lisboa, P.F., Ribeiro, M.P., Miguel, S.P., Simões, P.C., Correia, I.J., AguiarRicardo, A., 2014. Poly (vinyl alcohol)/chitosan asymmetrical membranes: highly controlled morphology toward the ideal wound dressing. J. Membr. Sci. 469, 262-271.

Morgado, P.I., Miguel, S.P., Correia, I.J., Aguiar-Ricardo, A., 2017. Ibuprofen loaded PVA/chitosan membranes: a highly efficient strategy towards an improved skin wound healing. Carbohydr. Polym. 159, 136-145.

Morgan, T.J., Morden, W.E., Al-muhareb, E., Herod, A.A., Kandiyoti, R., 2006. Essential oils investigated by size exclusion chromatography and gas chromatography - mass spectrometry. Energy Fuels 20, 734-737.

Najafi, M., Chery, J., Frey, M., 2018. Functionalized electrospun poly (vinyl alcohol) nanofibrous membranes with poly (methyl vinyl ether-alt-maleic anhydride) for protein adsorption. Materials 11, 1002.

Naseri, N., Algan, C., Jacobs, V., John, M., Oksman, K., Mathew, A.P., 2014. Electrospun chitosan-based nanocomposite mats reinforced with chitin nanocrystals for wound dressing. Carbohydr. Polym. 109, 7-15.

Negut, I., Grumezescu, V., Grumezescu, A., 2018. Treatment strategies for infected wounds. Molecules 23, 2392.
Nolting, D., Aziz, E.F., Ottosson, N., Faubel, M., Hertel, I.V., Winter, B., 2007. pH-induced protonation of lysine in aqueous solution causes chemical shifts in X-ray photoelectron spectroscopy. J. Am. Chem. Soc. 129, 14068-14073.

Oliveira, S.M., Alves, N.M., Mano, J.F., 2014. Cell interactions with superhydrophilic and superhydrophobic surfaces. J. Adhes. Sci. Technol. 28, 843-863.

Pereira, R.F., Barrias, C.C., Granja, P.L., Bartolo, P.J., 2013. Advanced biofabrication strategies for skin regeneration and repair. Nanomedicine 8, 603-621.

Pérez, D.J., Díaz-Reval, M.I., Obledo-Benicio, F., Zakai, U.I., Gómez-Sandoval, Z., RazoHernández, R.S., West, R., Sumaya-Martínez, M.T., Pineda-Urbina, K., RamosOrganillo, Á., 2017. Silicon containing ibuprofen derivatives with antioxidant and anti-inflammatory activities: an in vivo and in silico study. Eur. J. Pharmacol. 814, $18-27$.

Pires, L.R., Guarino, V., Oliveira, M.J., Ribeiro, C.C., Barbosa, M.A., Ambrosio, L., Pêgo, A.P., 2016. Ibuprofen-loaded poly (trimethylene carbonate-co-e-caprolactone) electrospun fibres for nerve regeneration. J. Tissue Eng. Regener. Med. 10, E154-E166.

Predoi, D., Iconaru, S., Buton, N., Badea, M., Marutescu, L., 2018. Antimicrobial activity of new materials based on lavender and basil essential oils and hydroxyapatite. Nanomaterials 8, 291.

Rajangam, K., Behanna, H.A., Hui, M.J., Han, X., Hulvat, J.F., Lomasney, J.W., Stupp, S.I., 2006. Heparin binding nanostructures to promote growth of blood vessels. Nano Lett. 6, 2086-2090.

Sahputra, I.H., Alexiadis, A., Adams, M.J., 2019. Effects of moisture on the mechanical properties of microcrystalline cellulose and the mobility of the water molecules as studied by the hybrid molecular mechanics-molecular dynamics simulation method. J. Polym. Sci., Part B: Polym. Phys.

Sakkas, H., Economou, V., Gousia, P., Bozidis, P., Sakkas, V., Petsios, S., Mpekoulis, G., Ilia, A., Papadopoulou, C., 2018. Antibacterial efficacy of commercially available essential oils tested against drug-resistant gram-positive pathogens. Applied Sciences 8, 2201.

Santos, C., Silva, C.J., Büttel, Z., Guimarães, R., Pereira, S.B., Tamagnini, P., Zille, A., 2014. Preparation and characterization of polysaccharides/PVA blend nanofibrous membranes by electrospinning method. Carbohydr. Polym. 99, 584-592.

Saviano, M., Aquino, R.P., Del Gaudio, P., Sansone, F., Russo, P., 2019. Poly (vinyl alcohol) 3D printed tablets: the effect of polymer particle size on drug loading and process efficiency. Int. J. Pharm. 561, 1-8.

Shamloo, A., Sarmadi, M., Aghababaie, Z., Vossoughi, M., 2018. Accelerated full-thickness wound healing via sustained bFGF delivery based on a PVA/chitosan/gelatin hydrogel incorporating PCL microspheres. Int. J. Pharm. 537, 278-289.

Sidgwick, G., McGeorge, D., Bayat, A., 2015. A comprehensive evidence-based review on the role of topicals and dressings in the management of skin scarring. Arch. Dermatol. Res. 307, 461-477.

Simões, D., Miguel, S.P., Correia, I.J., 2018a. Biofunctionalization of electrospun poly (caprolactone) fibers with Maillard reaction products for wound dressing applications. React. Funct. Polym. 131, 191-202.

Simões, D., Miguel, S.P., Ribeiro, M.P., Coutinho, P., Mendonça, A.G., Correia, I.J., 2018b. Recent advances on antimicrobial wound dressing: a review. Eur. J. Pharm. Biopharm. 127, 130-141.

Son, W.K., Youk, J.H., Lee, T.S., Park, W.H., 2004. The effects of solution properties and polyelectrolyte on electrospinning of ultrafine poly (ethylene oxide) fibers. Polymer 45, 2959-2966.

Thakkar, S., Misra, M., 2017. Electrospun polymeric nanofibers: new horizons in drug delivery. Eur. J. Pharm. Sci. 107, 148-167.

Torres-Giner, S., Martinez-Abad, A., Gimeno-Alcañiz, J.V., Ocio, M.J., Lagaron, J.M., 2012. Controlled delivery of gentamicin antibiotic from bioactive electrospun polylactide-based ultrathin fibers. Adv. Eng. Mater. 14, B112-B122.

Tungprapa, S., Jangchud, I., Supaphol, P., 2007. Release characteristics of four model drugs from drug-loaded electrospun cellulose acetate fiber mats. Polymer 48 , 5030-5041.

Van Antwerpen, P., Neve, J., 2004. In vitro comparative assessment of the scavenging activity against three reactive oxygen species of non-steroidal anti-inflammatory drugs from the oxicam and sulfoanilide families. Eur. J. Pharmacol. 496, 55-61.

Wang, X., Zhang, H., Jing, H., Cui, L., 2015. Highly efficient labeling of human lung cancer cells using cationic poly-l-lysine-assisted magnetic iron oxide nanoparticles. Nano-Micro Lett. 7, 374-384.

Xu, R., Xia, H., He, W., Li, Z., Zhao, J., Liu, B., Wang, Y., Lei, Q., Kong, Y., Bai, Y., 2016 Controlled water vapor transmission rate promotes wound-healing via wound reepithelialization and contraction enhancement. Sci. Rep. 6, 24596.

Yang, S.-A., Jeon, S.-K., Lee, E.-J., Shim, C.-H., Lee, I.-S., 2010. Comparative study of the chemical composition and antioxidant activity of six essential oils and their components. Nat. Prod. Res. 24, 140-151.

Yap, P., Krishnan, T., Yiap, B., Hu, C., Chan, K.G., Lim, S., 2014. Membrane disruption and anti-quorum sensing effects of synergistic interaction between $L$ avandula angustifolia (lavender oil) in combination with antibiotic against plasmid-conferred multi-drug-resistant E scherichia coli. J. Appl. Microbiol. 116, 1119-1128.

Yu, D.G., Zhang, X.F., Shen, X.X., Brandford-White, C., Zhu, L.M., 2009. Ultrafine ibuprofen-loaded polyvinylpyrrolidone fiber mats using electrospinning. Polym. Int. 58, 1010-1013.

Zamani, M., Prabhakaran, M.P., Ramakrishna, S., 2013. Advances in drug delivery via electrospun and electrosprayed nanomaterials. Int. J. Nanomed. 8, 2997.

Zeng, J., Xu, X., Chen, X., Liang, Q., Bian, X., Yang, L., Jing, X., 2003. Biodegradable electrospun fibers for drug delivery. J. Control. Release 92, 227-231.

Zhai, M., Yoshii, F., Kume, T., Hashim, K., 2002. Syntheses of PVA/starch grafted hydrogels by irradiation. Carbohydr. Polym. 50, 295-303.

Zhao, L., Mitomo, H., Zhai, M., Yoshii, F., Nagasawa, N., Kume, T., 2003. Synthesis of antibacterial PVA/CM-chitosan blend hydrogels with electron beam irradiation. Carbohydr. Polym. 53, 439-446. 